\title{
INFALL COLLAPSE SOLUTIONS IN THE INNER LIMIT: RADIATION PRESSURE AND ITS EFFECTS ON STAR FORMATION
}

\author{
Jasmin Jijina and Fred C. Adams \\ Department of Physics \\ University of Michigan, Ann Arbor, MI 48109 \\ and \\ Institute for Theoretical Physics \\ University of California, Santa Barbara, CA 93106 \\ submitted to The Astrophysical Journal: 18 July 1995
}

revised: 1 November 1995

\begin{abstract}
In this paper, we study infall collapse solutions for star formation in the small radius limit where the particle orbits become nearly pressure-free. We generalize previous solutions to simultaneously include the effects of both radiation pressure and angular momentum. The effects of radiation pressure can be modeled using a modified potential; for representative cases of such potentials, we obtain analytical solutions for the density and velocity fields. In general, radiation pressure limits the maximum mass of a forming star by reversing the infall when the star becomes sufficiently large. Our results imply that this maximum mass scale is given by the condition that the turnaround radius $R_{R}$ (the radius at which the radiation pressure force exceeds the gravitational force) exceeds the centrifugal radius $R_{C}$ (the angular momentum barrier). The maximum mass scale for a star forming within a rotating collapse flow with radiation pressure depends on the initial conditions, but is generally much larger than for the case of spherical infall considered previously. In particular, stars with masses $M_{*} \sim 100 M_{\odot}$ can form for a fairly wide range of initial conditions.
\end{abstract}

Subject headings: stars: formation - hydrodynamics 


\section{INTRODUCTION}

In this paper, we study generalized aspects of the collapse of molecular cloud cores to form stars. In particular, we include the effects of radiation pressure for the inner regime of the collapse flow. This work thus helps extend the current theory of star formation to include the formation of stars of higher mass.

In the current theory of star formation, molecular cloud cores provide the initial conditions for the star formation process. Star formation occurs when these cores collapse. A pressure supported star forms at the center of the collapse flow and a circumstellar disk forms around it from the infalling material with higher specific angular momentum (cf. the review of Shu, Adams, \& Lizano 1987). Thus, during this formative stage, a protostar consists of a central star/disk system which is deeply embedded within an infalling envelope of dust and gas. The characteristics of this infalling envelope largely determine the manner in which the protostar evolves.

Previous collapse calculations have focused on isothermal cloud cores which are either perfectly spherical (Larson 1969ab; Shu 1977; Hunter 1977) or slowly rotating (Cassen \& Moosman 1981; Terebey, Shu, \& Cassen 1984). These solutions have been extremely useful in studies of low-mass protostars. In particular, these infall solutions have been used as a starting point for radiative transfer calculations to determine the spectral energy distributions of protostellar objects; the results are in good agreement with observed protostellar candidates (e.g., Adams, Lada, \& Shu 1987, hereafter ALS; Butner et al. 1991; Kenyon, Calvet, \& Hartmann 1993). However, with large amounts of new kinematic data becoming available, more detailed collapse solutions are desirable.

These previous studies have shown that the collapse flow will remain nearly spherical in the outer regions, i.e., outside a centrifugal radius $R_{C}$ which is determined by conservation of angular momentum. When magnetic fields are present, an analogous magnetic barrier occurs at the radius $R_{B}$ where the Lorentz force of the magnetic field exceeds that of gravity (see Galli \& Shu 1993ab). In this present work, we study the collapse solutions in the inner regime $\left(r \sim R_{C}\right)$, where the infalling particles follow nearly ballistic trajectories as they spiral into the central star/disk system (Cassen \& Moosman 1981; Terebey et al. 1984). Previous work in this regime has been limited to the case of zero energy orbits and the gravitational potential of a point mass (the star).

In this work, we consider the additional effect of radiation pressure, which is important for protostellar objects with mass $M \geq 2 M_{\odot}$. In the current star formation scenario, there is an "opacity gap" immediately surrounding the star; here, the dust evaporates and radiation streams freely through this region. At the dust evaporation radius (often denoted as the "dust destruction front"), the ultraviolet and visible photons are absorbed in a thin shell and the photons are thermalized. Outside this radius, the infalling envelope is optically thick at infrared wavelengths where the warm dust reradiates. The gradient of this infrared radiation provides a radiation pressure which acts to decelerate the infalling gas.

Radiation pressure has been considered previously for spherical collapse (Larson \& Starrfield 1971; Kahn 1974; Wolfire \& Cassinelli 1986, 1987). These studies show that 
radiation pressure severely restricts the possible masses of forming stars. Nakano (1989) has argued that nonspherical infall can lead to the formation of more massive stars (see also Nakano, Hasegawa, \& Norman 1995); this work assumes both a flattened density distribution and a nonspherical radiation field. In this present study, we carry this idea further by finding a self-consistent infall-collapse solution which includes the effects of radiation pressure in the inner regime where rotation plays a large role and the flow is highly non-spherical. In our work, we assume a spherical radiation field and then calculate the corresponding nonspherical density field. The "inner" solutions resulting from this study can then be matched onto the "outer solutions" obtained elsewhere (e.g., Shu 1977; Adams et al. 1995) to provide a complete collapse solution for the star formation problem.

This paper is organized as follows. In $\S 2$, we formulate the collapse problem including the effects of radiation pressure and solve for the infall solution in the inner regime. In the next section ( $\S 3)$, we find the mass and radial scales in the problem; in particular, we find the maximum mass of a star/disk system that can form within this infall scenario. If disk accretion is efficient, this mass scale is the maximum stellar mass that can be assembled in the presence of radiation pressure. We consider several additional issues in $\S 4$, including the effects of magnetic fields. We conclude in $\S 5$ with a summary and discussion of our results.

\section{INFALL WITH RADIATION PRESSURE}

In this section, we determine the most important effects of radiation pressure on the infall collapse solutions. For the cases of interest, the inner limit of the collapse flow approaches a ballistic (pressure-free) form. We expect our solutions to be valid within the following range of (radial) size scales:

$$
R_{*}<r \sim R_{C} \ll r_{H}
$$

In this ordering constraint, the scale $R_{*}$ is the radius of the forming star and defines the inner boundary of the collapse flow. The scale $R_{C}$ is the centrifugal radius (defined more precisely below) which very roughly divides the nearly spherical outer region of the flow from the highly nonspherical inner region. Finally, the scale $r_{H}$ is the head of the expansion wave which divides the static outer core from the collapsing inner core (see Shu 1977). For radii comparable to the expansion wave radius $r_{H}$, pressure effects cannot be neglected and the solutions found here must be modified.

\subsection{Coupling of the Radiation Field and the Infalling Gas}

The coupling between the radiation field and the infalling material is determined primarily by the dust opacity $\kappa_{\nu}$, which specifies the cross section of the interaction. We are thus assuming that the dust and gas are themselves well coupled dynamically. This assumption should be well satisfied in the dense inner regions of interest here. In this regime, the radial force $f$ per unit mass exerted on the infalling gas by the radiation field is given by

$$
f=\int_{0}^{\infty} \frac{\kappa_{\nu} L_{\nu}}{4 \pi c r^{2}} d \nu=\frac{L}{4 \pi c} \frac{\kappa_{E}(T)}{r^{2}}
$$


where $L_{\nu}=4 \pi r^{2} F_{\nu}$ and $F_{\nu}$ is the flux density of the radiation field. The second equality essentially defines the weighted mean opacity $\kappa_{E}$ and is thus formally exact. We can "evaluate" this force term by approximating the weighted mean opacity as the Planck mean opacity, i.e.,

$$
f \approx \frac{L}{4 \pi c} \frac{\kappa_{P}(T)}{r^{2}} \approx \frac{L}{4 \pi c} \frac{\kappa_{P}\left(T_{0}\right)}{r^{2}} \frac{T}{T_{0}},
$$

where $T_{0}$ is a fiducial temperature. The use of the Planck mean opacity implicitly assumes that the protostellar radiation field is thermalized to the local dust temperature; this condition is satisfied in the optically thick inner regions of the collapsing envelope where essentially all of the effects of radiation pressure take place. In the second approximate equality, we have made the additional assumption that the Planck mean opacity is a linear function of temperature; this result is exact for the particular case in which $\kappa_{\nu} \propto \nu$, which is a good approximation for the frequencies (wavelengths) in the near infrared (Draine \& Lee 1984) where most of the interaction between the radiation field and the dust takes place.

Notice that we have assumed a spherical radiation field. This assumption is important because it allows for a completely analytical treatment of the problem. For high mass stars, the stellar component of the radiation field is nearly spherical and dominates the (nonspherical) radiation field of the disk. However, the nonspherical density distribution and the effects of bipolar outflows make the radiation field depart from spherical symmetry; these effects are not included in this present work.

We must determine the temperature distribution for protostellar envelopes. To a reasonable degree of approximation, the temperature profile in protostellar envelopes can be taken to be a power-law over the range of radial scales of interest. Simple analytical estimates show that the power-law index $q$ of the temperature distribution has the value $q \sim 5 / 6$ in the optically thick inner regions and flattens out to the range $q \sim 1 / 3-2 / 5$ in the optically thin outer regions (Adams \& Shu 1985). Thus, as a starting approximation to span both regimes, we adopt the simple form

$$
T(r)=T_{0}\left(r / r_{0}\right)^{-1 / 2}
$$

This simple form allows us to obtain analytic solutions which elucidate the basic physics of this problem. Once we have this basic understanding, we can study the effects of different forms for the temperature distribution.

Putting all of the above results together, we obtain an outward radial force due to radiation pressure of the form

$$
f=\frac{L}{4 \pi c} \frac{\kappa_{P}\left(T_{0}\right) \sqrt{r_{0}}}{r^{5 / 2}}
$$

Using this form for the radiation pressure force, we can derive an effective potential. The total potential (including both radiation pressure and gravity) can be written in the form

$$
V_{\mathrm{eff}}=\frac{G M}{r}\left\{\alpha r^{-1 / 2}-1\right\}
$$


where we have defined a parameter $\alpha$,

$$
\alpha \equiv \frac{L \kappa_{P}\left(T_{0}\right)}{6 \pi G M c} \sqrt{r_{0}}
$$

which has units of [length $]^{1 / 2}$. For the sake of definiteness, we take the reference radius $r_{0}$ to be the radius $r_{d}$ of the dust destruction front, i.e., the radius at which the temperature becomes larger than the sublimation temperature of dust grains. For typical interstellar dust grains, the Planck mean opacity $\kappa_{P}$ at this temperature $\left(T_{0}=T_{d} \sim 2300 \mathrm{~K}\right)$ is about $30 \mathrm{~cm}^{2} / \mathrm{g}$; for the sake of definiteness, we adopt this value throughout this paper. We can thus write the parameter $\alpha$ in the form

$$
\alpha \approx 1.6 \times 10^{-3} \sqrt{r_{d}}\left[\frac{\widetilde{L}}{\widetilde{M}}\right]
$$

where we have defined $\widetilde{L} \equiv L /\left(1 L_{\odot}\right)$ and $\widetilde{M} \equiv M /\left(1 M_{\odot}\right)$. Thus, as expected, for solar type stars, the correction to the orbits due to radiation pressure is small. Radiation pressure begins to play a substantial role in the infall when $\alpha^{2}>r_{d}$; this condition is met for $\widetilde{L} / \widetilde{M}>640$, which corresponds to moderately massive stars with $M \sim 7 M_{\odot}$.

\subsection{Orbital Solutions}

Given the potential, we can find the orbital solutions for parcels of gas falling in towards the central star. Our goal is to derive the functional forms for the velocity fields and the density profile resulting from the collapse of slowly rotating cloud cores. This calculation generalizes the case studied previously (Terebey, Shu, \& Cassen 1984; Cassen \& Moosman 1981; Ulrich 1976), where the inner collapse solution is derived using only the gravitational potential of the star itself.

Since the potential is spherically symmetric, the motion is confined to a plane and the angular momentum is conserved. This orbital plane can be described by the coordinates $(r, \phi)$ where the radius $r$ is the same in both the plane and the usual spherical coordinates. The angle $\phi$ is the angle in the plane is related to the usual spherical coordinates by the relation

$$
\cos \phi=\frac{\cos \theta}{\cos \theta_{0}}
$$

where $\theta_{0}$ is the angle of the asymptotically radial streamline (see below). We also assume orbits with zero total energy. For the case of no radiation pressure $(\alpha=0)$, the orbits are simply parabolas in the $(r, \phi)$ plane.

For the general case, we can integrate the equations of motion to obtain the solution for the orbits in the form

$$
\frac{\mu}{\mu_{0}} \cos \widetilde{\phi}+\left[1-\frac{\mu^{2}}{\mu_{0}^{2}}\right]^{1 / 2} \sin \widetilde{\phi}=\frac{2\left[\zeta r^{1 / 2}\left(1-\mu_{0}^{2}\right)+\alpha\right]^{2}}{2 \zeta r\left(1-\mu_{0}^{2}\right)+\alpha^{2}}-1 .
$$

The quantity $\mu_{0}$ is the cosine of the angle $\theta_{0}$ of the asymptotically radial streamline (i.e., the fluid trajectory that is currently passing through the position given by $\zeta$ and $\mu \equiv \cos \theta$ 
initially made the angle $\theta_{0}$ with respect to the rotation axis). The quantity $\zeta$ is defined by

$$
\zeta \equiv \frac{j_{\infty}^{2}}{G M r}=\frac{R_{C}}{r}
$$

where $j_{\infty}$ is the specific angular momentum of parcels of gas currently arriving at the origin along the equatorial plane. We have followed previous authors in assuming an initial state that is rotating at a constant rotation rate $\Omega$, so that the quantity $j_{\infty}$ is given by

$$
j_{\infty}=\Omega r_{\infty}^{2}
$$

where $r_{\infty}$ is the starting radius of the material that is arriving at the origin at a given time. This radius can be determined by inverting the initial mass distribution $M(r)$, which we discuss below. Finally, the angle $\widetilde{\phi}$ arises from the constant of integration and is defined by

$$
\cos \widetilde{\phi}=\frac{\alpha^{2}-2 R_{C}\left(1-\mu_{0}^{2}\right)}{\alpha^{2}+2 R_{C}\left(1-\mu_{0}^{2}\right)} .
$$

Using these results, the "orbit equation" can be "simplified" to the form

$$
\left[\alpha^{2}-2 \zeta r\left(1-\mu_{0}^{2}\right)\right]\left[\frac{\mu}{\mu_{0}}-1\right]+\left[1-\frac{\mu^{2}}{\mu_{0}^{2}}\right]^{1 / 2} \alpha \sqrt{8 \zeta r}\left(1-\mu_{0}^{2}\right)^{1 / 2}=2 \zeta^{2} r\left(1-\mu_{0}^{2}\right)^{2}+4 \alpha \zeta r^{1 / 2}\left(1-\mu_{0}^{2}\right) .
$$

The effects of radiation pressure on the infalling orbits can be illustrated as in Figure 1 , which shows projected orbits in the meridional plane for varying amounts of radiation pressure. Notice that as the radiation pressure (and hence $\alpha$ ) increases, the orbits become increasingly deflected.

When the orbits begin to turn around, adjacent orbits will intersect each other and the gaseous material will shock. The locus of this shock surface (or "turn around surface") is shown in Figure 2 for varying amounts of radiation pressure. Notice that adjacent streamlines do not intersect each other on the way in.

\subsection{The Centrifugal Radius}

The centrifugal radius $R_{C}$ plays an important role in determining the nature of the infall solution. To evaluate $R_{C}$, we must invert the mass distribution of the initial state. Here, we find $R_{C}$ for the two types of initial states which are most applicable for star formation.

We first consider the case of a molecular cloud core described by an isothermal equation of state. We thus obtain the profile

$$
M(r)=\frac{2 a^{2} r}{G} \quad \text { and hence } \quad r_{\infty}=\frac{G M}{2 a^{2}} .
$$

Thus, for isothermal initial conditions, the centrifugal radius $R_{C}$ can be written

$$
R_{C}=\frac{\Omega^{2} G^{3} M^{3}}{16 a^{8}}
$$


where we have combined equations [2.10], [2.11], and [2.14a].

We are also interested in infall solutions which apply to the formation of more massive stars where isothermal initial conditions may not apply. Observations indicate that in regions of molecular clouds with higher mass clumps, the molecular line widths show a substantial nonthermal component (e.g., Larson 1985; Myers \& Fuller 1992). Motivated by the finding that these observed molecular linewidths decrease with density according to the law $\Delta v \propto \rho^{-1 / 2}$, one can derive a "logatropic" equation of state $P=P_{0} \log \rho$ to describe the fluid (see Lizano \& Shu 1989; Adams et al. 1995). For this case, the equilibrium mass distribution and the corresponding $r_{\infty}$ are given by

$$
M(r)=\left[\frac{2 \pi P_{0}}{G}\right]^{1 / 2} r^{2} \quad \text { and hence } \quad r_{\infty}=M^{1 / 2}\left[\frac{2 \pi P_{0}}{G}\right]^{-1 / 4}
$$

where $P_{0}$ is the pressure scale that determines the amount of nonthermal support in the cloud. The centrifugal radius for logatropic initial conditions is given by

$$
R_{C}=\frac{\Omega^{2} M}{2 \pi P_{0}}
$$

We note that in the presence of radiation pressure, the effective centrifugal radius becomes modified, i.e., an effective centrifugal radius can be defined (see equation [2.29]).

\subsection{Velocity and Density Fields}

Given the orbital solution, we can find the velocity fields and the corresponding density distribution. We put together the geometrical relation [2.8], conservation of angular momentum, and conservation of energy,

$$
\frac{1}{2}\left(v_{r}^{2}+v_{\theta}^{2}+v_{\varphi}^{2}\right)=\frac{G M}{r}-\alpha \frac{G M}{r^{3 / 2}},
$$

to determine the velocity field, which can be written in the form

$$
\begin{gathered}
v_{r}=-\left(\frac{G M}{r}\right)^{1 / 2}\left\{2-2 \alpha r^{-1 / 2}-\zeta\left(1-\mu_{0}^{2}\right)\right\}^{1 / 2}, \\
v_{\theta}=\left(\frac{G M}{r}\right)^{1 / 2}\left\{\frac{1-\mu_{0}^{2}}{1-\mu^{2}}\left(\mu_{0}^{2}-\mu^{2}\right) \zeta\right\}^{1 / 2}, \\
v_{\varphi}=\left(\frac{G M}{r}\right)^{1 / 2}\left(1-\mu_{0}^{2}\right)\left(1-\mu^{2}\right)^{-1 / 2} \zeta^{1 / 2}
\end{gathered}
$$

Notice that $\zeta, \mu$, and $\mu_{0}$ are related by the orbit equation [2.13] so that the velocity field is in fact completely determined for any position $(r, \theta)$. 
The density distribution of the infalling material can be obtained by applying conservation of mass along a streamtube (Terebey, Shu, \& Cassen 1984; Chevalier 1983), i.e.,

$$
\rho(r, \theta) v_{r} r^{2} \sin \theta d \theta d \varphi=-\frac{\dot{M}}{4 \pi} \sin \theta_{0} d \theta_{0} d \varphi_{0} .
$$

In this present context, we ignore the fact that some particle orbits can turn around and leave the system. Thus, this solution for the density is valid for radii larger than the turn around radius given by equation [3.2] below. Combining the above equations allows us to write the density profile in the form

$$
\rho(r, \theta)=\frac{\dot{M}}{4 \pi v_{r} r^{2}} \frac{d \mu_{0}}{d \mu} .
$$

The properties of the collapsing core determine the form of $d \mu_{0} / d \mu$. In the present case, the orbit equation [2.13] determines the form of $d \mu_{0} / d \mu$; thus, with the radial velocity given by equation [2.17], the density field is completely specified (analytically, but implicitly).

The density distribution as a function of angle and for varying amounts of radiation pressure is shown in Figure 3. Two trends are evident from this figure: For a given radius, the density decreases with decreasing angle and with increasing values of the radiation pressure constant $\alpha$ for small values of $r / R_{C}$.

\subsection{Equivalent Spherical Envelope}

One way to characterize the effects of both angular momentum and radiation pressure is to define an equivalent spherical density distribution. In other words, we take the angular average of the nonspherical density distribution (see Adams \& Shu 1986; ALS). Since one effect of both rotation and radiation pressure is to prevent material from falling to smaller radii, this equivalent spherical density distribution will not be equal to the density distribution for spherical (nonrotating) initial conditions. We note that this approach has proven to be a useful characterization of protostellar envelopes for purposes of determining their spectral energy distributions (ALS; Kenyon et al. 1993). The equivalent spherical density distribution is given by

$$
\langle\rho\rangle=\int_{0}^{\pi / 2} \rho(r, \theta) \sin \theta d \theta=C r^{-3 / 2} \mathcal{A}(r),
$$

where the constant $C$ is defined by

$$
C \equiv \frac{\dot{M}}{4 \pi \sqrt{2 G M}}
$$

and where we have defined an asphericity function $\mathcal{A}(r)$,

$$
\mathcal{A}(r) \equiv \int_{0}^{1} d \mu \frac{d \mu_{0}}{d \mu}\left\{1-\alpha r^{-1 / 2}-\frac{1}{2} \zeta\left(1-\mu_{0}^{2}\right)\right\}^{-1 / 2} .
$$


Notice that the density distribution in the spherical limit is given by $\rho=C r^{-3 / 2}$ (see Shu 1977) so that the effects of rotation and radiation pressure are incorporated into the asphericity function $\mathcal{A}(r)$.

We can evaluate the integral appearing in equation [2.24] by changing the integration variable from $\mu$ to $\mu_{0}$ and changing the lower end of the range of integration from 0 to a critical value $\mu_{C}$. The difference in the range of integration arises because streamlines from all initial angles cannot fall to arbitrarily small radii. For large radii, streamlines from all initial angles (i.e., all values of $\mu_{0}$ ) are represented. Inside the centrifugal barrier $R_{C}$, however, only streamlines originating preferentially from the poles can reach these smaller radii (although for $\alpha \neq 0$, no streamlines will reach radii $r<\alpha^{2}$ ). The last streamline that can reach a given radius for a given $\alpha$ is given by $\mu_{C}$; clearly $\mu_{C} \rightarrow 0$ as $r \rightarrow \infty$ and $\mu_{C} \rightarrow 1$ as $r \rightarrow 0$. After evaluating the integral, we find

$$
\mathcal{A}(r)=\left(\frac{2}{\zeta}\right)^{1 / 2} \log \left[\frac{1+(2 / \zeta)^{1 / 2}\left(1-\alpha r^{-1 / 2}\right)^{1 / 2}}{\mu_{C}+\left[(2 / \zeta)\left(1-\alpha r^{-1 / 2}\right)+\mu_{C}{ }^{2}-1\right]^{1 / 2}}\right]
$$

The asphericity function $\mathcal{A}(r)$ is shown in Figure 4 for different amounts of radiation pressure. As the radiation pressure is increased, the cusp near the centrifugal radius becomes sharper and is also pushed back to larger radii. Notice also that for $\alpha>0$, a second "cusp" forms at the location of the shock surface (as shown in Figure 2).

We can explicitly evaluate the asphericity function in the limits of large and small radii. For large radii, all of the streamlines reach $r$ and hence $\mu_{C}=0$. For this case, we have

$$
\mathcal{A}(r)=\left(\frac{2}{\zeta}\right)^{1 / 2} \log \left[\frac{1+(2 / \zeta)^{1 / 2}\left(1-\alpha r^{-1 / 2}\right)^{1 / 2}}{\left[(2 / \zeta)\left(1-\alpha r^{-1 / 2}\right)-1\right]^{1 / 2}}\right]
$$

Thus, in the limit $r \rightarrow \infty$, we have $\mathcal{A}(r) \rightarrow 1$. In other words, as expected, the departure of the solution from the pure spherical case disappears in the limit of large radii. In the opposite limit of small radii, the result depends on the relative size of the centrifugal radius $R_{C}$ and the radial scale $\alpha^{2}$ set by radiation pressure. Under the ordering

$$
\alpha^{2} \ll r \ll R_{C}
$$

we obtain the result

$$
\mathcal{A}(r) \approx(2-\sqrt{2}) \frac{r}{R_{C}}
$$

which is identical to the case of no radiation pressure (see ALS). Thus, the density distribution has the form $\rho \sim r^{-1 / 2}$ at the intermediate spatial scales given by equation [2.27]. Notice that we need not consider the other limit in which $\alpha^{2} \geq r$ because all orbits will turn around before reaching the radius $r$.

The above discussion implies that there is an effective centrifugal radius in this problem. This radius, denoted here as $R_{C R}$, is the smallest radius reached by streamlines 
from all initial angles (all values of $\mu_{0}$ ). This radius is easily calculated from the orbit solution and is given by

$$
R_{C R}=R_{C}\left[1-\alpha R_{C}^{-1 / 2}(1-\sqrt{2} / 2)\right]^{-2}
$$

Notice that, as expected, the effective centrifugal radius is larger than for the case with no radiation pressure $(\alpha=0)$.

\subsection{Matching onto Outer Solutions}

In the limit of large radius, this inner solution for the collapse flow matches smoothly onto the general class of spherical infall solutions which have a well defined mass infall rate; the isothermal collapse solution (Shu 1977) is a well known example. In this limit, the orbit equation [2.13] shows that $\mu \rightarrow \mu_{0}$ and the velocity field becomes purely radial with $v_{r} \rightarrow-(2 G M / r)^{1 / 2}$ (see equations [2.17-2.19]). Thus, in the limit of large $r$, the density field becomes simply $\rho=C r^{-3 / 2}$, with the constant $C$ defined by equation [2.23]. In other words, the density distribution approaches the form appropriate for spherical free-fall with a given (not necessarily constant) mass infall rate.

In order for the large radius limit to apply, the radius $r$ must be large compared to both the centrifugal radius $R_{C}$ and the radial scale $R_{R}=\alpha^{2}$ set by radiation pressure. Thus, this solution approaches the "large radius limit" slightly slower than the case of rotation only. However, since we generally expect $\alpha^{2} \ll R_{C}$, this effect is small.

\section{MAXIMUM MASS SCALES FOR FORMING STARS}

In this section, we discuss the maximum mass of a star that can be formed through a rotating infall flow which includes radiation pressure. In the absence of radiation pressure, most of the infalling material falls directly onto a circumstellar disk. Although disk physics is not well understood, stability considerations suggest that a substantial fraction of this mass will eventually accrete onto the central star. When radiation pressure is important, some particle orbits will be "turned away" before they impact the disk or the star. As radiation pressure increases, less and less of the infalling material actually hits the disk. When the radiation pressure reaches a critical level, none of the infalling material can reach the disk and the star/disk system effectively becomes isolated from its environment. In this section, we calculate the mass scale at which this isolation occurs for several cases of interest. If disk accretion is efficient, then this mass scale is the maximum stellar mass that can be formed within this infall scenario.

\subsection{Mass Limits for the Infall Collapse Solution}

Given the full solution for the particle orbits in the previous section, we can determine conditions for which material hits the disk and the corresponding conditions for which material becomes turned away by radiation pressure. We first note that parcels of gas will hit the disk when they pass through the equatorial plane, i.e., when $\mu=0$. Using 
the orbit equation, we can solve for the radius at which the particles hit the disk; this condition can be written in the form

$$
R_{C}\left(1-\mu_{0}^{2}\right) r^{-1 / 2}=R_{C}^{1 / 2}\left(1-\mu_{0}^{2}\right)^{1 / 2}+\alpha(\sqrt{2} / 2-1) .
$$

Similarly, using the solution for the velocity field, we can determine that radius at which orbits will turn around due to the radiation pressure; this condition can be written in the form

$$
R_{C}\left(1-\mu_{0}^{2}\right) r^{-1 / 2}=-\alpha+\left[\alpha^{2}+2 R_{C}\left(1-\mu_{0}^{2}\right)\right]^{1 / 2}
$$

Note that, as formulated, the orbits will always turn around for some sufficiently small radius. This behavior is simply a reflection of the fact that the repulsive term in the potential (due to radiation pressure) always becomes larger than the gravitational attraction for sufficiently small radius. In practice, however, when the "turn around radius" becomes smaller than the radius of the dust destruction front, parcels of gas will not actually turn around.

Given the expressions [3.1] and [3.2] for the radii at which infalling parcels of gas hit the disk and turn around, respectively, we can write down a condition which must be met in order for parcels of gas to hit the disk before they reach the turn around radius. This condition can be written in the form

$$
\alpha^{2}<2 R_{C}\left(1-\mu_{0}^{2}\right)
$$

Alternately, we can use this condition to determine the range of initial angles which lead to orbits which intersect the disk. Formally, all particle orbits will hit the disk provided that

$$
\mu_{0}^{2}<1-\alpha^{2} / 2 R_{C}<1
$$

Thus, when

$$
\alpha^{2}>2 R_{C}
$$

then none of the orbits will hit the disk plane and all of the orbits will turn around. In other words, when $\alpha$ becomes greater than $2 R_{C}$, the infall flow onto the central star/disk system effectively stops. As a result, the condition [3.5] defines the maximum mass of a star that can form through this infall scenario. This condition makes intuitive sense: The quantity $\alpha^{2}$ defines the radius at which the radiation pressure dominates over gravity; the centrifugal radius $R_{C}$ defines the radius at which angular momentum dominates over gravity. In order for radiation pressure to dominate over angular momentum, some condition like equation [3.5] is necessary.

In order to evaluate the maximum mass scale, we must evaluate the coupling parameter $\alpha$ (see equation [2.7]). Thus, we must find the dust destruction radius as a function of the stellar luminosity. We find that

$$
r_{d}=\left[\frac{L}{16 \pi \sigma T_{d}^{4}} \frac{\kappa_{*}}{\kappa_{P}\left(T_{d}\right)}\right]^{1 / 2} \equiv R_{D} \widetilde{L}^{1 / 2} \approx 5.7 \times 10^{11} \mathrm{~cm} \widetilde{L}^{1 / 2}
$$


where $\widetilde{L}$ is the total luminosity in units of $L_{\odot}$ and where $\kappa_{*}$ is the opacity of the material to stellar photons (see Stahler, Shu, \& Taam 1980 and Appendix B of Adams 1990). We have defined a fiducial value $R_{D}$ of the dust destruction radius which is that appropriate for the luminosity of the sun. Notice that the dust grains absorb stellar photons with an efficiency proportional to $\kappa_{*}$ and emit photons with a lower efficiency proportional to $\kappa_{P}\left(T_{d}\right)$. Thus, the dust grain temperature at any given radius is larger than the naive "planet approximation" by the factor $\left[\kappa_{*} / \kappa_{P}\left(T_{d}\right)\right]^{1 / 4} \sim 1.6$, where we have adopted $\kappa_{*}$ $=200 \mathrm{~cm}^{2} / \mathrm{g}$.

Using the expression [3.6] for the dust destruction radius, we can evaluate the coupling parameter $\alpha$ and then find the maximum mass scale. We first consider the case of isothermal initial conditions, since most previous work on star formation has begun with the collapse of an isothermal cloud core; however, the alternate "logatropic" initial state described below might be more applicable to the formation of high mass stars. For the isothermal case, after considerable rearrangement, we can write the condition [3.5] for radiation pressure to halt infall onto the disk in the form

$$
L^{5 / 2} M^{-5} \geq 2[3 \pi c \Omega]^{2} G^{5} a^{-8}\left[\kappa_{P}\left(T_{d}\right)\right]^{-3 / 2} \kappa_{*}^{-1 / 2}\left[\pi \sigma T_{d}^{4}\right]^{1 / 2}
$$

Inserting numerical values and rearranging once again, we find

$$
\widetilde{L} \widetilde{M}^{-2} \geq 1570 \widetilde{\Omega}^{4 / 5} a_{35}^{-16 / 5}
$$

where we have defined $\widetilde{\Omega} \equiv \Omega /\left(1 \mathrm{~km} \mathrm{~s}^{-1} \mathrm{pc}^{-1}\right)$ and $a_{35} \equiv a /\left(0.35 \mathrm{~km} \mathrm{~s}^{-1}\right)$. The relation [3.7] implies a maximum mass of a forming star for a given set of the initial conditions.

Notice that the mass $M$ appearing in these constraints is the total mass that has fallen onto the star/disk system. In general, only a fraction of this mass becomes part of the star so that we should write $M_{*}=\gamma M$. However, disk stability considerations (Adams, Ruden, \& Shu 1989; Shu et al. 1990) show that $\gamma \geq 2 / 3$; otherwise, the disk would be violently unstable to self-gravitating perturbations. Thus, the difference between $M$ and $M_{*}$ is not overly large.

In order to evaluate this limit, we must use some type of mass/luminosity relation. The stars of interest are generally in the mass range of $20-100 M_{\odot}$ so that their evolutionary time scales are short compared to the infall time scale. This ordering implies that the stars will have (nearly) their main sequence configurations while they are still in the infall phase of evolution (see, e.g., Yorke \& Krügel 1977; Yorke 1979; Palla \& Stahler 1990). We can thus use a main sequence mass/luminosity relation for this present discussion (e.g., Ezer \& Cameron 1967; Allen 1976; Phillips 1994).

Our results for an isothermal collapse are shown in Figure 5 where the mass limit is given as a function of the initial conditions $a$ and $\Omega$. For a given mass, the region of parameter space to the lower right of the curve is excluded, i.e., a star of the given mass cannot form with initial conditions in that region. The most restrictive limit occurs for stars with the largest value of $L / M^{2}$ (see equation [3.7b]) which occurs for $M \approx 50 M_{\odot}$. Thus, if the rotation rate is large enough and/or the effective sound speed is low enough, then the luminosity never becomes large enough to halt the infall (in other words, the left 
hand side of equation [3.7b] never exceeds the right hand side). In this case, radiation pressure alone does not restrict the mass of a forming star.

Next, we consider the case of logatropic initial conditions. In this case, the condition for radiation pressure to halt the infall can be written in the form

$$
L^{5 / 2} M^{-3} \geq \frac{16}{\pi P_{0}}[3 \pi c G \Omega]^{2}\left[\kappa_{P}\left(T_{d}\right)\right]^{-3 / 2} \kappa_{*}^{-1 / 2}\left[\pi \sigma T_{d}^{4}\right]^{1 / 2} .
$$

After inserting numbers and rearranging, we obtain

$$
\widetilde{L} \widetilde{M}^{-6 / 5} \geq 1.1 \times 10^{4} \widetilde{\Omega}^{4 / 5} \widetilde{P}^{-2 / 5}
$$

where $\widetilde{\Omega}$ is defined as before and where we have also defined $\widetilde{P} \equiv P_{0} /\left(3.6 \times 10^{-11}\right.$ dyne $\mathrm{cm}^{-2}$ ); this numerical value for the pressure scale corresponds to the magnetic pressure $B^{2} / 8 \pi$ for a field strength of $30 \mu \mathrm{G}$. In order to evaluate this limit, we can once again use the main sequence mass/luminosity relationship.

The mass limit as a function of logatropic initial conditions is shown in Figure 6. As before, for a given mass, the region of parameter space to the lower right of the curve is excluded, i.e., a star of the given mass cannot form with initial conditions in that region. Also, the quantity $\widetilde{L} \widetilde{M}^{-6 / 5}$ has a maximum value close to $10^{4}$; thus, if the pressure scale $P_{0}$ is sufficiently small and/or the rotation rate is sufficiently large, then radiation pressure alone cannot restrict the mass of the forming star. Notice also that the mass limits for these logatropic initial conditions are generally less restrictive than the isothermal case shown in the previous figure. In other words, for the expected range of pressure scales, a lower rotation rate is required to allow material to fall to sufficiently large radii in the disk to evade the effects of radiation pressure.

As shown above, the maximum mass of a forming star increases with increasing rotation rate $\Omega$ and decreases with increasing pressure of the initial state (given by either the effective sound speed $a$ or the pressure scale $P_{0}$ ). This behavior has a straightforward physical interpretation: When the rotation rate is larger, the angular momentum is larger, and infalling material tends to fall to larger radii in order to conserve angular momentum. As a result, material does not fall as far down the potential well and cannot be affected (as much) by radiation pressure. When the sound speed (or the pressure scale) for the initial configuration is larger, the infall rate $\dot{M}$ is larger, and material falls farther down the potential well for a given rotation rate; at these smaller radii, radiation pressure has a greater effect. The difference between the isothermal and logatropic initial conditions is also clear: The latter case has more material at larger radii in the initial equilibrium state and hence the effects of angular momentum conservation are greater for a given rotation rate; this effect, in turn, makes the mass limits weaker for the logatropic case.

\subsection{Mass Limit for Spherical Infall}

For comparison, we write down the maximum mass scale for the case of spherical infall (no rotation). In this case, the parcels of gas and dust fall inward radially through the potential well given by equation [2.6]. The orbits will "turn around" if the coupling term 
$\alpha r^{-1 / 2}$ becomes larger than unity before the parcel reaches the dust destruction radius (where the coupling vanishes). Thus, the condition for radiation pressure to dominate is given by the relation

$$
\alpha^{2} \geq r_{d}
$$

where the dust destruction radius is given by equation [3.6] above. Notice that the condition for reversing spherical infall is generally much weaker than the condition for reversing the rotating infall (because, in general, $R_{C} \gg r_{d}$ ). Thus, the maximum mass scale for the spherical case will be much lower than the more realistic rotating case found above. After some rearrangement, we can write this condition in the form

$$
\frac{L}{M} \geq \frac{6 \pi G c}{\kappa_{P}\left(T_{d}\right)} .
$$

Using numerical values, we find

$$
\frac{\widetilde{L}}{\widetilde{M}} \geq 640 \text {. }
$$

This condition has the following simple physical interpretation. For sufficiently large $\widetilde{L} / \widetilde{M}$, the specific work done on the infalling gas by the radiation field exceeds the depth of the gravitational potential well at $r_{d}$ (see ALS). This value is essentially equivalent to that found by Kahn (1974), although he used a higher value for the dust destruction temperature $(3670 \mathrm{~K})$ and found a corresponding higher value for the maximum mass (see also Wolfire \& Cassinelli 1987 for further discussion).

\subsection{General Mass Limits}

We have shown that the condition for radiation pressure to reverse infall onto a star/disk system is that the turnaround radius $R_{R}$ of the radiation pressure must be larger than the centrifugal radius $R_{C}$ of the rotating infall. In order words, the condition

$$
R_{R}>\eta R_{C}
$$

defines the maximum mass of a forming star; the dimensionless constant $\eta$ is of order unity, but its exact value must be determined from the true infall solution. In this subsection, we use this result to study the effects of varying the different parameters in the problem. In particular, we find the maximum mass of a forming star for different types of coupling between the radiation field and the infalling gas and for different initial conditions in the cloud core.

We first define a radiation pressure "structure constant" $\delta_{R}$ through the relation

$$
\delta_{R} \equiv \frac{L_{\odot} \kappa_{P}\left[T_{d}\right]}{4 \pi G c M_{\odot}} \approx \frac{1}{430}
$$

Thus, $\delta_{R}$ is an intrinsically small parameter in the problem. 
We want to consider different power-law forms for the radiation pressure force (see equation $[2.5]$ ). We can write this force in the form

$$
f=\frac{G M}{r^{2}} \delta_{R}(\widetilde{L} / \widetilde{M})\left(\frac{r_{d}}{r}\right)^{q}
$$

where $q$ is an arbitrary power-law index which we expect to lie in the range $1 / 3 \leq q \leq 1$. The effective turnaround radius $R_{R}$, where radiation pressure dominates gravity, is given by

$$
R_{R}=\left[\delta_{R} \widetilde{L} / \widetilde{M}\right]^{1 / q} r_{d}
$$

In order to isolate the effects of different power-law indices $q$, we specify the initial state to be in logatropic equilibrium so that the centrifugal radius is given by equation [2.15]. We can write this radius in the form

$$
R_{C}=R_{0} \widetilde{M} \widetilde{\Omega}^{2} \widetilde{P}^{-1} \approx 9 \times 10^{15} \mathrm{~cm} \widetilde{M} \widetilde{\Omega}^{2} \widetilde{P}^{-1}
$$

where $\widetilde{M}, \widetilde{\Omega}$, and $\widetilde{P}$ are defined as before. The radius $R_{0}$ is the "natural value" of the centrifugal radius.

For this case, the mass limit can be written in the form

$$
\left[\delta_{R} \widetilde{L} / \widetilde{M}\right]^{1 / q} \widetilde{L}^{1 / 2} \widetilde{M}^{-1}>\eta \frac{R_{0}}{R_{D}} \widetilde{\Omega}^{2} \widetilde{P}^{-1} \approx 1.5 \times 10^{4} \eta \widetilde{\Omega}^{2} \widetilde{P}^{-1}
$$

For a given set of initial conditions, the right hand side of this equation is a (generally large) constant. The left hand side is an increasing function of the mass, although it saturates at sufficiently large masses. As the index $q$ increases, the mass scale given by equation [3.16] also increases. In other words, a larger mass star is needed to reverse the infall. This result is expected physically: As the index $q$ increases, the effective range of the radiation pressure force decreases (see equation [3.13]) and hence radiation has a smaller effect on the infall.

We also want to determine the effects of different initial conditions on the maximum mass scale for forming stars. As discussed in $\S 2$, we expect the initial equilibrium states for star formation to be described by equations of state which are isothermal or softer. As a result, the initial mass profile should be of the form $M \sim r^{b}$ where the index $b$ lies in the range $1 \leq b \leq 2$. This set of states implies a corresponding set of centrifugal radii of the form

$$
R_{C}=R_{0} \widetilde{M}^{d}
$$

where the index $d$ lies in the range $1 \leq d \leq 3$ (see equations [2.14] and [2.15]). The fiducial radius $R_{0}$ must be close to the disk radius for solar type stars, i.e., $R_{0} \sim 100$ AU. The mass limit for forming stars can then be written in the form

$$
\left[\delta_{R} \widetilde{L} / \widetilde{M}\right]^{1 / q} \widetilde{L}^{1 / 2} \widetilde{M}^{-d}>\eta \frac{R_{0}}{R_{D}}
$$


Typically, we expect the right hand side of this constraint to be a large number, $10^{3}-10^{5}$. As the index $d$ increases, the constraint becomes harder to satisfy, i.e., a larger mass star is required to reverse the infall.

\section{ADDITIONAL ISSUES}

In this section, we briefly and heuristically discuss several additional issues which are relevant to infall collapse solutions of the type considered in this paper. In particular, we discuss the leading order effects of magnetic fields, a critical value of the mass infall rate, and the manner in which rotation places additional constraints on the infall solution and the corresponding maximum mass scales of forming stars.

\subsection{Magnetic Fields}

Thus far in this discussion, we have ignored the effects of magnetic fields on the collapse. However, since molecular clouds are supported on large scales by magnetic fields (Zuckerman \& Palmer 1974; Shu et al. 1987) and since cloud cores are most likely formed through the process of magnetic field diffusion (e.g., Mouschovias 1978; Shu 1983; Nakano 1984; Lizano \& Shu 1989), magnetic fields should be incorporated into the collapse solution simultaneously with rotation and radiation pressure. We can get an order of magnitude estimate of the magnetic field strength required to affect the infall as follows. The effective strength of the field can be measured by the radius $R_{B}$ at which the perturbation in the meridional velocity produced by the Lorentz force is equal to the asymptotic free-fall velocity; this radius plays a role in the collapse flow similar to that of the centrifugal radius and thus defines the outer radius of a "Pseudodisk" (see Galli \& Shu 1993ab). The magnetic radius can be written

$$
R_{B}=k_{B}\left[\frac{G^{2} B^{4}}{a}\right]^{1 / 3} t^{7 / 3}
$$

where $k_{B}$ is a dimensionless constant. For the case of an isothermal collapse model, we can replace the time variable by the mass, i.e., $t=M / \dot{M}=M G / a^{3} m_{0}$. The magnetic radius is then given by

$$
R_{B}=k_{B}\left[\frac{G^{9} B^{4} M^{7}}{a^{22} m_{0}^{7}}\right]^{1 / 3}
$$

where $m_{0}=0.975$ is the dimensionless mass determined from the isothermal collapse solution (Shu 1977).

Since the magnetic radius plays an analogous role to the centrifugal barrier which we have already included in the problem, we can make the following rough argument: We expect radiation pressure to reverse the infall for a magnetically collapsing core when the turnaround radius $R_{R}$ exceeds the magnetic radius $R_{B}$. This condition can be written in the form

$$
L^{5 / 2} M^{-13 / 3} \geq \eta k_{B}[12 \pi c]^{2} G^{5}\left[\frac{B^{4}}{a^{22} m_{0}^{7}}\right]^{1 / 3}\left[\kappa_{P}\left(T_{d}\right)\right]^{-3 / 2} \kappa_{*}^{-1 / 2}\left[\pi \sigma T_{d}^{4}\right]^{1 / 2},
$$


where we have included a constant $\eta$ of order unity. After inserting numerical values, we can write this result in the form

$$
\widetilde{L} \widetilde{M}^{-26 / 15} \geq 1.9 \times 10^{4} \widetilde{B}^{8 / 15} a_{35}^{-44 / 15}
$$

where we have defined $\widetilde{B} \equiv B / 30 \mu \mathrm{G}$ and where we have set $\eta=1=k_{B}$ to obtain the numerical value. This relation defines the maximum mass of a forming star for a collapse flow which includes magnetic fields and radiation pressure. This maximum mass scale is a function of the initial conditions, defined here by the sound speed $a$ and the magnetic field strength $B$; the result is shown in Figure 7.

\subsection{Critical Mass Infall Rate}

The approximations of this paper become invalid when the mass infall rate becomes too small. In order for infall to occur, the mass infall rate must be large enough to produce a ram pressure greater than the radiation pressure at the dust destruction front. This condition can be written in the form

$$
\dot{M} v_{i n}>L / c
$$

where $v_{i n}$ is the speed of the infalling material evaluated at the radius $r_{d}$. This condition implies that for sufficiently high luminosity $L$, the radiation pressure will dominate the total ram pressure and will terminate the infall. However, this constraint is not overly restrictive. If we insert numerical values, the critical mass infall rate, denoted here as $\dot{M}_{C}$, becomes

$$
\dot{M}_{C}=1.4 \times 10^{-10} M_{\odot} \mathrm{yr}^{-1} \widetilde{L}^{5 / 4} \widetilde{M}^{-1 / 2}
$$

where we have used equation [3.6] to evaluate the dust destruction radius $r_{d}$. In order for infall to occur, the mass infall rate must exceed this critical rate. For example, for a massive star with $\widetilde{M}=100$, the luminosity is $\widetilde{L}=1.2 \times 10^{6}$ and the critical mass infall rate is $\dot{M}_{C}=6 \times 10^{-4} M_{\odot} \mathrm{yr}^{-1}$.

\subsection{Rotation and Time Constraints}

In obtaining the maximum masses of forming stars $(\S 3)$, we have assumed that infalling material which reaches the disk can eventually become part of the star through the process of disk accretion. Although disk physics is not completely understood, stability considerations suggest that this assumption is reasonable, provided that sufficient time is available for disk accretion to occur. In this section we show that the rather short lifetime of massive stars places an additional constraint on this process.

Conservation of angular momentum causes incoming material to fall initially onto a circumstellar disk rather than directly onto the star. In the absence of disk accretion, this effect shuts off the mass flow onto the star. However, even in the presence of disk accretion, angular momentum can eventually shut off the infall because the time scale for disk accretion to take place becomes longer than the lifetime of the star. Very roughly, the shortest time scale for a disk accretion process is the dynamical time scale at the 
outer disk edge. For a disk with radius $R_{C}$, the centrifugal radius, this time scale is roughly given by

$$
\tau^{2}=\frac{R_{C}^{3}}{G M}
$$

where we have assumed that the outer disk rotation curve is nearly Keplerian. If we now enforce the constraint that this time scale $\tau$ must be shorter than the lifetime $\tau_{*}$ of a massive star, we obtain a constraint on the centrifugal radius of the form

$$
R_{C} \leq \tau_{*}^{2 / 3}(G M)^{1 / 3}
$$

We note that this constraint is necessary but not sufficient. A very massive star can significantly disrupt a circumstellar disk and thereby disrupt disk accretion and evolution (see Hollenbach et al. 1994).

We can use the above result to obtain a maximum stellar mass constraint that is independent of the initial conditions. We have already shown that in order for radiation pressure not to reverse the infall, we must have $R_{R}=\alpha^{2}<2 R_{C}$. Thus, a star can continue to gain mass through the combination of infall and disk accretion only when following ordering is satisfied,

$$
\alpha^{2} / 2<R_{C}<\tau_{*}^{2 / 3}(G M)^{1 / 3}
$$

Thus, when the radiation turnaround radius $\left(R_{R} \sim \alpha^{2}\right)$ exceeds the critical radius defined by the right hand side of this inequality, the ordering constraint cannot be met for any set of initial conditions (for any collapse model, isothermal or otherwise). Thus, the condition for the maximum stellar mass can be written in the form

$$
L^{5 / 2} M^{-7 / 3}=2 \tau_{*}^{2 / 3} G^{7 / 3}[12 \pi c]^{2}\left[\kappa_{P}\left(T_{d}\right)\right]^{-3 / 2} \kappa_{*}^{-1 / 2}\left[\pi \sigma T_{d}^{4}\right]^{1 / 2}
$$

As usual, we can insert numerical values and rearrange this limit to obtain the form

$$
\widetilde{L} \widetilde{M}^{-14 / 15}=5.6 \times 10^{4} \tau_{6}^{4 / 15}
$$

where we have defined $\tau_{6} \equiv \tau_{*} /\left(10^{6} \mathrm{yr}\right)$. As a reference point, note that $\tau_{6} \approx 3.6$ for a star with mass $\widetilde{M}=100$ (Ezer \& Cameron 1967). The limit [4.9] represents the largest mass accessible to a star that forms within the collapse scenario considered here for any set of initial conditions. The left hand side of this equation is (almost) the luminosity to mass ratio in solar units; for massive main sequence in the mass range $100-200 M_{\odot}$, this ratio is approximately $1-2 \times 10^{4}$, i.e., slightly smaller than the numerical value on the right hand side. Thus, the maximum luminosity to mass ratio allowed for forming stars within this scenario is slightly larger than the $L / M$ ratios for very massive stars.

For completeness, we note that the time constraint considered here can be written as a mass limit which is independent of the effects of radiation pressure. For isothermal initial conditions, this limit can be written in the form

$$
M<\sqrt{8} G^{-1} \tau_{*}^{1 / 4} a^{3} \Omega^{-3 / 4}
$$


or, in terms of dimensionless quantities,

$$
\widetilde{M}<30 \widetilde{\Omega}^{-3 / 4} a_{35}^{3} \tau_{6}^{1 / 4}
$$

For a given set of initial conditions $a$ and $\Omega$, the mass of a star that can form through the combined process of infall and disk accretion is limited by the constraint of equation [4.10]. Notice that this limit only becomes restrictive for high mass stars because of the dependence on the stellar lifetime $\tau_{6}$.

Finally, we also note that there exists yet another a related constraint on the mass. In the original molecular cloud core, gaseous material at sufficiently large radii is centrifugally supported in the initial state. This radius is given by $R=a / \Omega$ for an isothermal cloud core. Thus, the total mass that is available to fall inward is given by

$$
M=\mathcal{F} \frac{2 a^{3}}{G \Omega}
$$

where we have used the mass profile for an isothermal cloud core and where we have introduced a factor $\mathcal{F}$ to take geometry into account. Gas located in the polar directions is not centrifugally supported and can thus fall inwards. We thus obtain

$$
\widetilde{M}=21 \mathcal{F} a_{35}^{3} \widetilde{\Omega}^{-1}
$$

The reason why these two constraints [4.10] and [4.11] are nearly identical is that the time scale for stellar evolution of massive stars (about $10^{6} \mathrm{yr}$ ) is about the same as the rotation period of a molecular cloud core. 


\section{SUMMARY AND DISCUSSION}

In this paper, we have generalized the infall collapse solution for star formation to include the effects of radiation pressure in the inner regime. Our results can be summarized as follows:

[1] We have shown the effects of radiation pressure can be modeled using a modified potential. The radiation field is expected to be nearly spherically symmetric and creates an outward force. Furthermore, the radial dependence of the radiation pressure force is expected to decrease with radius faster than the gravitational force. As a result, the effects of radiation pressure can be incorporated using a modified potential of the general form

$$
V_{\mathrm{eff}}=-\frac{G M}{r}\{1-g(r)\},
$$

where $g(r)$ is a decreasing function of radius $r$. We have used the approximation $g=$ $\alpha r^{-1 / 2}$ which corresponds to assuming that the coupling between the radiation field and the gas is proportional to the Planck mean opacity and that the temperature distribution has the simple form $T \sim r^{-1 / 2}$.

[2] We have found an analytic solution (equation [2.13]) for the zero energy orbits of the modified potential described above (see equation [2.6]). This solution provides the velocity field (equations [2.17 - 2.19]) and the density field (equation [2.21]) for infall collapse solutions which simultaneously include both angular momentum and radiation pressure.

[3] The infall solution has three important radial scales: The dust destruction radius $r_{d}$, the centrifugal radius $R_{C}$, and the radius $R_{R}=\alpha^{2}$ at which radial orbits are reversed by radiation pressure. The characteristics of the orbits and the infall solution are determined by the ratios of these three radial scales. In order for angular momentum to play a significant role in the collapse, the centrifugal radius $R_{C}$ must exceed the dust destruction radius $r_{d}$. Similarly, in order for radiation pressure to play an important role, the turnaround radius $R_{R}$ must exceed the dust destruction radius $r_{d}$. For the case $R_{C} \gg R_{R}$, radiation pressure perturbs the orbits so that infalling material hits the disk at larger radii; however, the qualitative nature of the infall is similar to the case of no radiation pressure. In the opposite limit $R_{R} \gg R_{C}$, the orbits turn around before they impact the disk and infall effectively ceases.

[4] This infall collapse solution implies a maximum mass scale for forming stars. For this solution, the condition for radiation pressure to halt the infall onto the central star/disk system is given by $\alpha^{2}=R_{R}>2 R_{C}$. The resulting maximum mass scale is a function of the initial conditions for protostellar collapse (see Figures 5 and 6). In general, the constraints on the masses of forming stars (see equations [3.7] and [3.8]) are considerably less restrictive for rotating collapse with radiation pressure than for the case of purely spherical infall (equation [3.10]). Our results show that massive stars with $M_{*} \sim 100 M_{\odot}$ can form for a wide range of initial conditions.

[5] We have argued that the constraint which limits infall onto the disk, $R_{R}>R_{C}$, is more robust than its derivation. As a result, we can use this condition to find the mass limits on forming stars for a wide range of initial conditions and for different assumptions 
about the coupling of the radiation field to the infalling gas (see §3.3). As the coupling of the radiation field falls off more quickly with radius, the effects of radiation pressure decrease, and stars of higher mass can form with a given set of initial conditions. Similarly, as the equation of state becomes softer, stars of higher mass can form. These statements are quantified by equations [3.16] and [3.18].

[6] We have explicitly found orbit solutions for different assumptions about the coupling between the radiation field and the infalling gas. In particular, we have considered the two limiting cases in which the strength of the coupling decreases as slowly and as rapidly as possible (see Appendix B).

[7] We have discussed the leading order effects of magnetic fields on a rotating collapse which includes radiation pressure. The net effect of magnetic fields is to prevent material from falling as far inwards (due to the Lorentz force). As a result, radiation pressure has less of an effect in the presence of magnetic fields.

[8] We have shown that this scenario for forming massive stars (through the combination of infall and disk accretion) breaks down when the natural time scale of the disk becomes longer than the lifetime of a massive star. This effect places a limit on the ratio of luminosity to mass $(L / M)$ for forming stars. Although this limit is independent of initial conditions, it is not very restrictive: Stars with masses in the range $100-200 M_{\odot}$ are allowed by a small margin (see equation [4.9] and $\S 4.3$ ).

In some sense, the results of this paper show that radiation pressure has less impact on the infall than previous studies (with spherical infall) have implied. The basic physical reason for this difference is that previous studies have not simultaneously included both angular momentum and radiation pressure. For the typical range of parameters applicable to protostellar collapse, angular momentum plays an extremely important role. In particular, the centrifugal radius $R_{C}$ (which determines the size of the angular momentum barrier) is much larger than the radius of the star or the dust destruction front. As a result, in order for radiation pressure to have a significant impact, it must affect infalling material at the (large) size scales of the centrifugal radius $R_{C}$.

The results of this paper can be tested observationally, although such tests are difficult. The collapse solution of this paper predicts that the infalling envelopes of forming massive stars will be highly evacuated for radii less than the turnaround radius $R_{R}$, and will have the general form as illustrated in Figures 2 and 3. Although the density distribution of circumstellar material is not directly measurable, its form can be deduced (or at least highly constrained) by comparing radiative transfer calculations with observed spectral energy distributions and emission maps. For high mass stars, some work along these lines has already been done (Churchwell, Wolfire, \& Wood 1990; see also Wolfire \& Churchwell 1994). The former study shows that the dust envelope around a newly formed O6 star is highly evacuated within $\sim 10^{17} \mathrm{~cm}$ of the star and that the density distribution must be fairly flat, i.e., the density must increase inwards less rapidly than $\rho \sim r^{-1 / 2}$. These results are in basic agreement with those of this paper. For this star (with mass $M=34 M_{\odot}$ and $L=2.5 \times 10^{5} L_{\odot}$ ), the turnaround radius $R_{R}=4 \times 10^{16} \mathrm{~cm}$ and the maximum centrifugal radius consistent with the stellar lifetime is $\sim 2 \times 10^{18} \tau_{6}^{2 / 3}$ cm. Thus, a circumstellar envelope with a fairly flat density distribution $\left(\rho \sim r^{-1 / 2}\right)$ in 
the range $r=10^{17}-10^{18} \mathrm{~cm}$ arises naturally from our collapse solution for massive stars.

Another testable prediction of this calculation is the presence of rather large amounts of mass $\left(10-100 M_{\odot}\right)$ entering the circumstellar disks associated with high mass stars. For this collapse solution, most of the infalling material falls directly onto the disk rather than directly onto the star. If the disk accretion rate is at all slower than the envelope infall rate, then material piles up to form a massive disk. Such massive disks will have bright dust continuum emission at millimeter and submillimeter wavelengths. In addition, the ionized (inner) edges of the disks will be bright centimeter wave sources. Existing studies have searched for massive disks associated with embedded protostars of low and intermediate mass; some studies provide supporting evidence for massive disks (e.g., Reipurth et al. 1993; Ho, Terebey, \& Turner 1994), whereas other studies suggest that massive disks are somewhat rare (e.g., Terebey, Chandler, \& André 1993; André \& Montmerle 1994).

Although this paper provides a significant generalization of the protostellar collapse problem, many unresolved issues still remain. This work has been limited to gravitational potentials which correspond to point masses, whereas realistic systems include both extended components (i.e., disks) and time varying components (i.e, binaries). These issues should be addressed in future work.

\section{Acknowledgements}

We would like to thank Frank Shu, Joel Tohline, and Rick Watkins for useful discussions. We also thank the referee - Sue Terebey - for many useful suggestions. This work was supported by an NSF Young Investigator Award, NASA Grant No. NAG 52869, and by funds from the Physics Department at the University of Michigan; we also thank the Institute for Theoretical Physics at U. C. Santa Barbara and NSF Grant No. PHY94-07194. 


\section{APPENDIX A: ALTERNATE FORM OF THE ORBIT EQUATION}

In this Appendix, we briefly describe another way to express the orbit equation. This form is useful for evaluating the various expressions found in the text. We first define

$$
x=\frac{\mu}{\mu_{0}},
$$

and

$$
A \equiv \frac{2\left[\zeta r^{1 / 2}\left(1-\mu_{0}^{2}\right)+\alpha\right]^{2}}{2 \zeta r\left(1-\mu_{0}^{2}\right)+\alpha^{2}}-1 .
$$

Notice that the quantity $A=1$ at the turnaround radius $\alpha^{2}=R_{R}$. The orbit equation can then be written in the form

$$
x=A \cos \widetilde{\phi}+\left[1-A^{2}\right]^{1 / 2} \sin \widetilde{\phi},
$$

where $\cos \widetilde{\phi}$ is defined by equation [2.12] in the text. The expression [A3] gives $\mu$ as a function of the initial value $\mu_{0}$. To obtain this form, we solved a quadratic equation which formally has two roots; the second root, which corresponds to using a minus sign in equation [A3], is not relevant. 


\section{APPENDIX B: LIMITING CASES FOR THE RADIATION PRESSURE APPROXIMATION}

In this Appendix, we assess the severity of the approximation used in this paper to model the effects of radiation pressure. We have assumed that the radiation pressure can be incorporated using the form for the coupling given by equation [2.2]. In order to evaluate this term, we have made two approximations. The first is that we can use the Planck mean opacity for the weighted mean opacity $\kappa_{E}$ and that the Planck mean opacity is linear in temperature. The second approximation is that we assume the temperature distribution has the simple power-law form $T \sim r^{-1 / 2}$ for the radii of interest in this problem. Thus, the coupling term $\sim r^{-1 / 2}$ in this approximation. In general, we can write the effective potential in the form

$$
V_{\text {eff }}=-\frac{G M}{r}\{1-g(r)\}
$$

where $g(r)$ is an unspecified dimensionless function which describes the radial variation of the radiation pressure term relative to the gravitational potential. We expect that the function $g(r)$ will vary faster than a constant $(g=$ constant corresponds to a constant ratio of radiation pressure to gravity) but will vary less quickly than $g \sim r^{-1}$. The case considered in the text has the intermediate behavior $g \sim r^{-1 / 2}$.

As a start, we can solve for the orbits in the two limiting cases which bracket the expected behavior for the radiation pressure term. In order to solve the orbit equation, we must solve the integral,

$$
J[g]=\Delta \phi=\gamma^{1 / 2} \int_{r}^{\infty} \frac{d r}{r}[2 r(1-g)-\gamma]^{-1 / 2}
$$

where $\gamma=R_{C}\left(1-\mu_{0}^{2}\right)$ is proportional to the square of the specific angular momentum of a parcel of gas beginning at angle $\theta_{0}$. In the first case, where the coupling term varies slowly as possible, we take $g=$ constant and obtain the solution

$$
\zeta\left(1-\mu_{0}^{2}\right)=\left(1-\mu / \mu_{0}\right)(1-g) .
$$

This solution corresponds to reducing the gravitational constant by the factor $(1-g)$. In the opposite limit, where the coupling varies as quickly as possible, we write the function $g$ in the form $g=\xi / r$. After some algebra, this solution can be written in the form

$$
1-\cos \left[\phi(1+2 \xi / \gamma)^{1 / 2}\right]=\frac{2 \xi+\gamma}{r},
$$

where $\cos \phi=\mu / \mu_{0}$. For any case, the orbital behavior should be bracketed by the solutions given by equations [B3] and [B4].

We now consider another way to look at the problem of different radial dependences for the coupling term $g(r)$. If we change variables according to

$$
x^{2} \equiv \frac{\gamma}{2 r},
$$


the integral $J[g]$ can be written in the form

$$
J[g]=2 \int_{0}^{x_{e}} d x\left[1-g-x^{2}\right]^{-1 / 2},
$$

where we have taken the limits of integration to be spatial infinity (where $r \rightarrow \infty$ and $x \rightarrow 0)$ and $x_{e}=\sqrt{2} / 2$, which corresponds to the radial position $(r=\gamma)$ at which a parcel of gas hits the disk in the case of no radiation pressure $(g=0)$. We want to determine the effects of relatively small departures from the case of no radiation pressure. We thus take the first variation of the functional $J[g]$ about the "point" $g=0$ to obtain

$$
\delta J=\int_{0}^{x_{e}} d x g\left[1-x^{2}\right]^{-3 / 2} .
$$

We now consider a class of models for $g$ of the form

$$
g=\beta\left(x / x_{e}\right)^{n}
$$

where the index $n$ is confined to the range $0-2$ (which corresponds to the expected radial behavior of $g(r)$ as described above). We can evaluate the first variation $\delta J$ for the various models of the coupling given by equation [B8]. We find that $\delta J / \beta=1, \sqrt{2}-1$, and $1-\pi / 4$ for the cases $n=0,1$, and 2 , respectively. Thus, the exact form of the coupling does not greatly change the size of the action for a given value of $\beta$. In other words, the functional form of the coupling $g$ only changes the value of the orbit integral by a factor of two, provided that the coupling function has sufficiently non-pathological behavior (such as that given by equation [B8]).

\section{REFERENCES}

Adams, F. C. 1990, ApJ, 363, 578

Adams, F. C., Galli, D., Najita, J., Lizano, S., \& Shu, F. H. 1995, ApJ, in preparation

Adams, F. C., Lada, C. J., \& Shu, F. H. 1987, ApJ, 312, 788 (ALS)

Adams, F. C., Ruden, S. P., \& Shu, F. H. 1989, ApJ, 347, 959

Adams, F. C., \& Shu, F. H. 1985, ApJ, 296, 655

Adams, F. C., \& Shu, F. H. 1986, ApJ, 308, 836

André, P., \& Montmerle, T. 1994, ApJ, 420, 837

Allen, C. W. 1976, Astrophysical Quantities (London: Athlone Press)

Butner, H. M., Evans, N. J., Lester, D. F., Levreault, R. M., \& Strom, S. E. 1991, ApJ, 376, 676 
Cassen, P., \& Moosman, A. 1981, Icarus, 48, 353

Chevalier, R. 1983, ApJ, 268, 753

Churchwell, E., Wolfire, M. G., \& Wood, D.O.S. 1990, ApJ, 354, 247

Draine, B. T., \& Lee, H. M. 1984, ApJ, 285, 89

Ezer, D., \& Cameron, A.G.W. 1967, Canadian J. Phys., 45, 3429

Galli, D., \& Shu, F. H. 1993a, ApJ, 417, 220

Galli, D., \& Shu, F. H. 1993b, ApJ, 417, 243

Ho, P. T., Terebey, S., \& Turner 1994, ApJ, 423, 320

Hollenbach, D., Johnstone, D., Lizano, S., \& Shu, F. 1994, ApJ, 428, 654

Hunter, C. 1977, ApJ, 218, 834

Kahn, F. D. 1974, A \& A, 37, 149

Kenyon, S. J., Calvet, N., \& Hartmann, L. 1993, ApJ, 414, 676

Larson, R. B. 1969a, MNRAS, 145, 271

Larson, R. B. 1969b, MNRAS, 145, 297

Larson, R. B., \& Starrfield, S. 1971, A \& A, 13, 190

Larson, R. B. 1985, MNRAS, 214, 379

Lizano, S., \& Shu, F. H. 1989, ApJ, 342, 834

Mouschovias, T. Ch. 1978, in Protostars and Planets, ed. T. Gehrels (Tucson: University of Arizona Press), p. 209

Myers, P. C., \& Fuller, G. A. 1992, ApJ, 396, 631

Nakano, T. 1984, Fund. Cosmic Phys., 9, 139

Nakano, T. 1989, ApJ, 345, 464

Nakano, T., Hasegawa, T., \& Norman, C. 1995, ApJ, in press

Palla, F., \& Stahler, S. W. 1990, ApJ, 360, L47

Phillips, A. C. 1994, The Physics of Stars (Chichester: Wiley)

Reipurth, B., Chini, R., Krügel, E., Kreysa, E., \& Sievers, A. 1993, A \& A, 273, 221

Shu, F. H. 1977, ApJ, 214, 488

Shu, F. H. 1983, ApJ, 273, 202

Shu, F. H., Adams, F. C., \& Lizano, S. 1987, A R A \& A, 25, 23

Shu, F. H., Tremaine, S., Adams, F. C., \& Ruden, S. P. 1990, ApJ, 358, 495

Stahler, S. W., Shu, F. H., \& Taam R. E. 1980, ApJ, 241, 637

Terebey, S., Chandler, C. J., \& André, P. 1993, ApJ, 414, 759

Terebey, S., Shu, F. H., \& Cassen, P. 1984, ApJ, 286, 529 
Ulrich, R. K. 1976, ApJ, 210, 377

Wolfire, M. G., \& Cassinelli, J. 1986, ApJ, 310, 207

Wolfire, M. G., \& Cassinelli, J. 1987, ApJ, 319, 850

Wolfire, M. G., \& Churchwell, E. 1994, ApJ, 427, 889

Yorke, H. W. 1979, A \& A, 80, 308

Yorke, H. W., \& Krügel, E. 1977, A \& A, 54, 183

Zuckerman, B., \& Palmer, P. 1974, A R A \& A, 12, 279

\section{FIGURE CAPTIONS}

Figure 1. Effects of radiation pressure on an infalling trajectory in the meridional plane. Solid curves show the projected orbits for a given initial angle $\theta_{0}$ and for varying values of radiation pressure; shown here are curves for $\alpha^{2} / R_{C}=0.25,0.50$, and 1.0. The dotted curve shows the orbit for the case of no radiation pressure $\alpha=0$. The star is located at the origin of the coordinates. (a) Initial angle $\theta_{0}=\pi / 4\left(\mu_{0}=\sqrt{2} / 2\right)$. (b) Initial angle $\theta_{0}=\pi / 8$.

Figure 2. The "turn around surface" for varying amounts of radiation pressure. The dotted curves show the surfaces at which orbits are reversed due to radiation pressure. The different curves correspond to varying amounts of radiation pressure given by $\alpha^{2} / R_{C}$ $=0.25-2.0$ in increments of 0.25 . The solid curves show representative trajectories (orbits) with initial angles $\theta_{0}$ in increments of $\pi / 16$ (for the case with $\alpha^{2} / R_{C}=0.75$ ).

Figure 3. Density profiles including radiation pressure. The various curves show the density as a function of radius for angles $\theta=0, \pi / 6, \pi / 4$, and $\pi / 3$. The horizontal axis shows the radius in units of the centrifugal radius $R_{C}$; the vertical axis shows the density scaled to the value $\rho_{C}$, which is the density distribution from the spherical solution evaluated at the centrifugal radius $\left(\rho_{C}=C R_{C}^{-3 / 2}\right)$. (a) Density distribution for $\alpha^{2} / R_{C}$ $=0$. (b) Density distribution for $\alpha^{2} / R_{C}=0.5$. (c) Density distribution for $\alpha^{2} / R_{C}=$ 1.0 .

Figure 4. The asphericity function $\mathcal{A}(r)$ for varying amounts of radiation pressure. The function $\mathcal{A}$ is the ratio of the spherically averaged density profile $\langle\rho\rangle$ to the density profile obtained for purely spherical collapse, i.e., $\mathcal{A}=\langle\rho\rangle r^{3 / 2} C^{-1}$. The radius (plotted on the horizontal axis) is given in units of the centrifugal radius $R_{C}$. The different curves are for varying amounts of radiation pressure with $\alpha^{2} / R_{C}=0,0.1,0.3$, and 0.5 .

Figure 5. Maximum mass of a forming star for purely isothermal collapse. This figure shows the plane of initial conditions, i.e., the sound speed $a$ constitutes the horizontal 
axis and the rotation rate $\Omega$ constitutes the vertical axis. The labeled curves show the maximum mass of a star that can form with the given values of the initial conditions. The region in the upper left part of the diagram has no upper mass limit.

Figure 6. Maximum mass of a forming star for purely logatropic collapse. This figure shows the plane of initial conditions, i.e., the pressure scale $P_{0}$ constitutes the horizontal axis and the rotation rate $\Omega$ constitutes the vertical axis. The labeled curves show the maximum mass of a star that can form with the given values of the initial conditions. The region in the upper left part of the diagram has no upper mass limit.

Figure 7. Maximum mass of a forming star for an isothermal collapse flow which includes radiation pressure and magnetic fields. This figure shows the plane of initial conditions, i.e., the sound speed $a$ constitutes the horizontal axis and magnetic field strength $B$ constitutes the vertical axis. The labeled curves show the maximum mass of a star that can form with the given values of the initial conditions. The region in the upper left part of the diagram has no upper mass limit. 


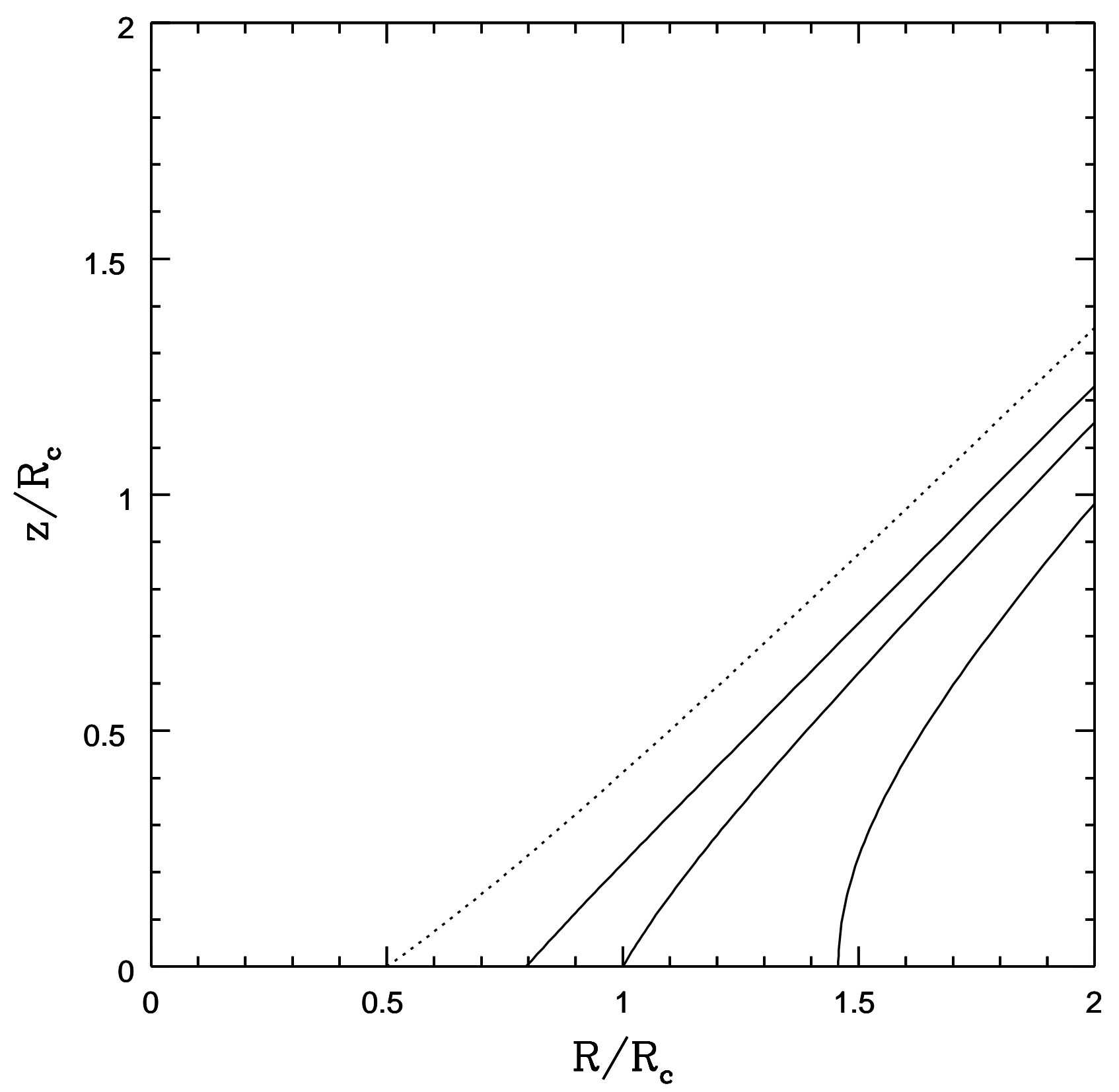




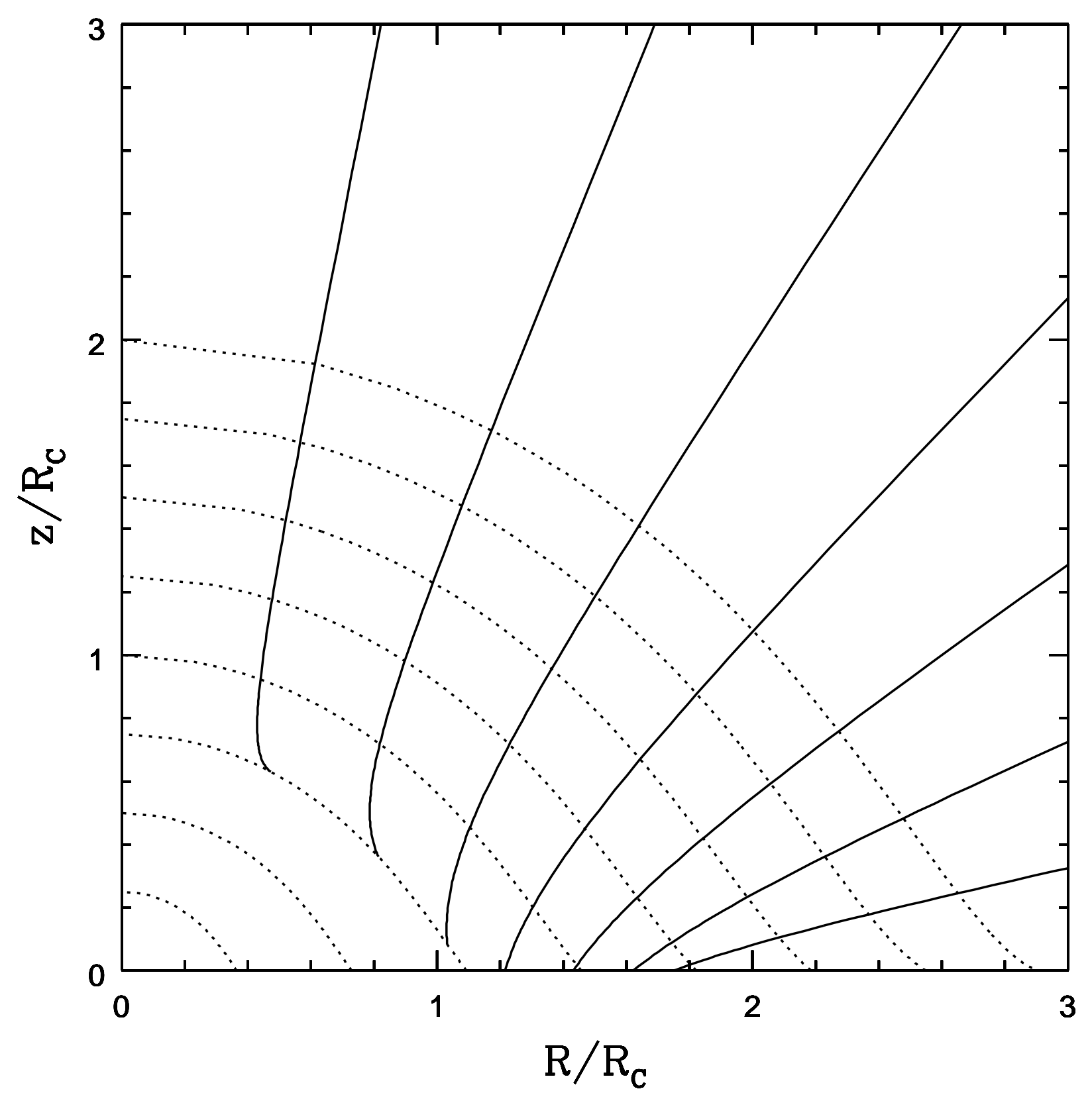




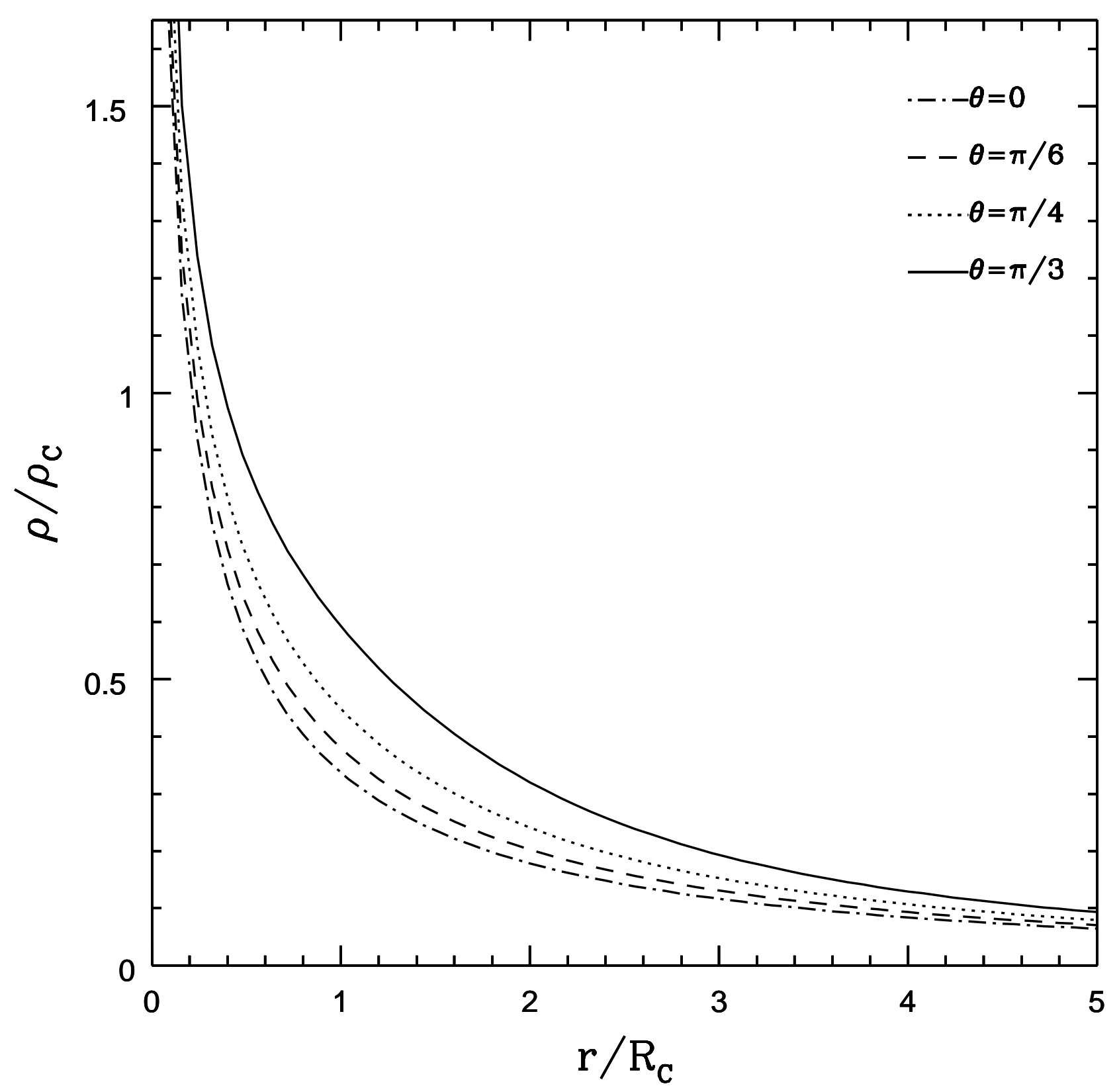




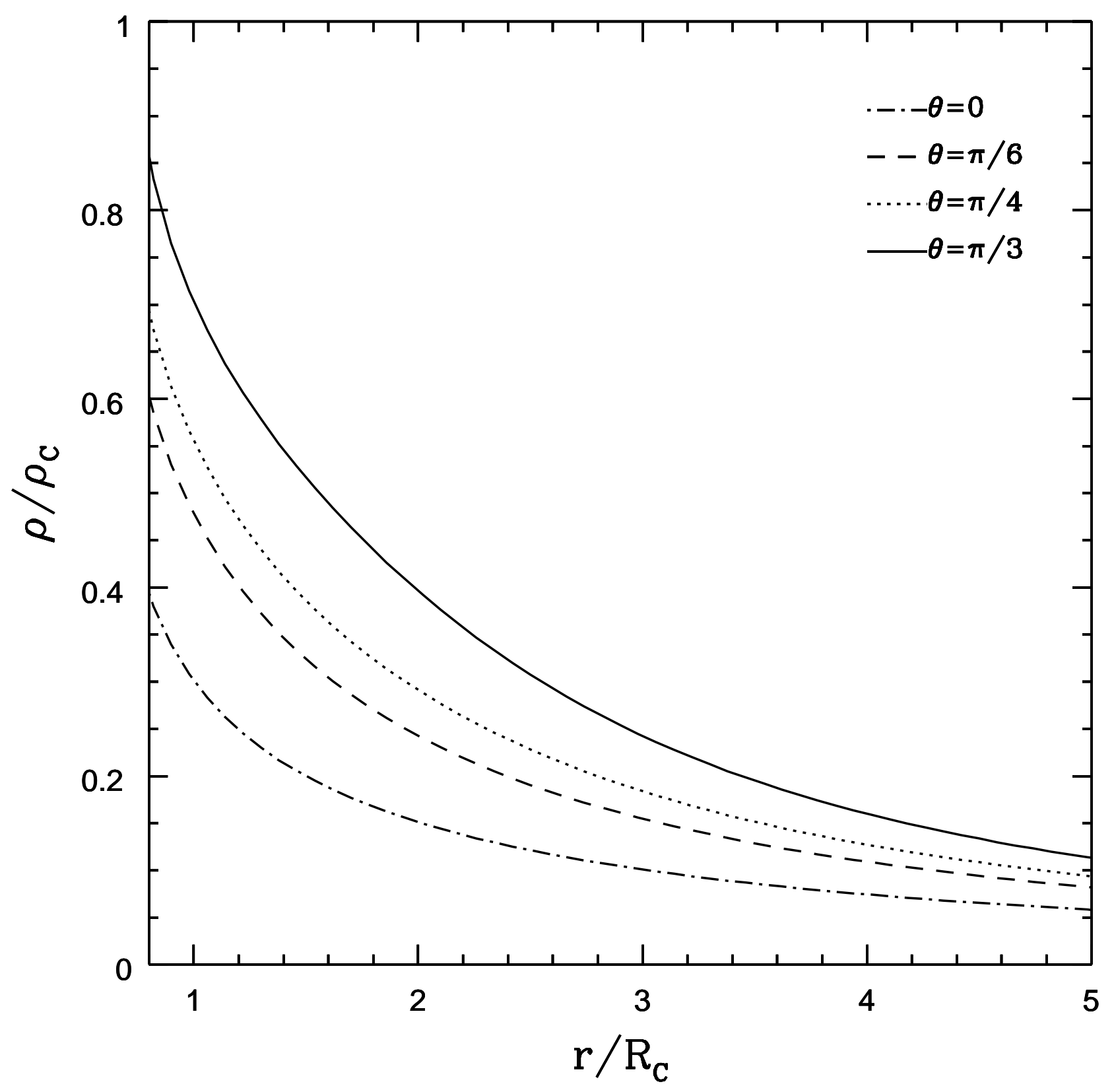




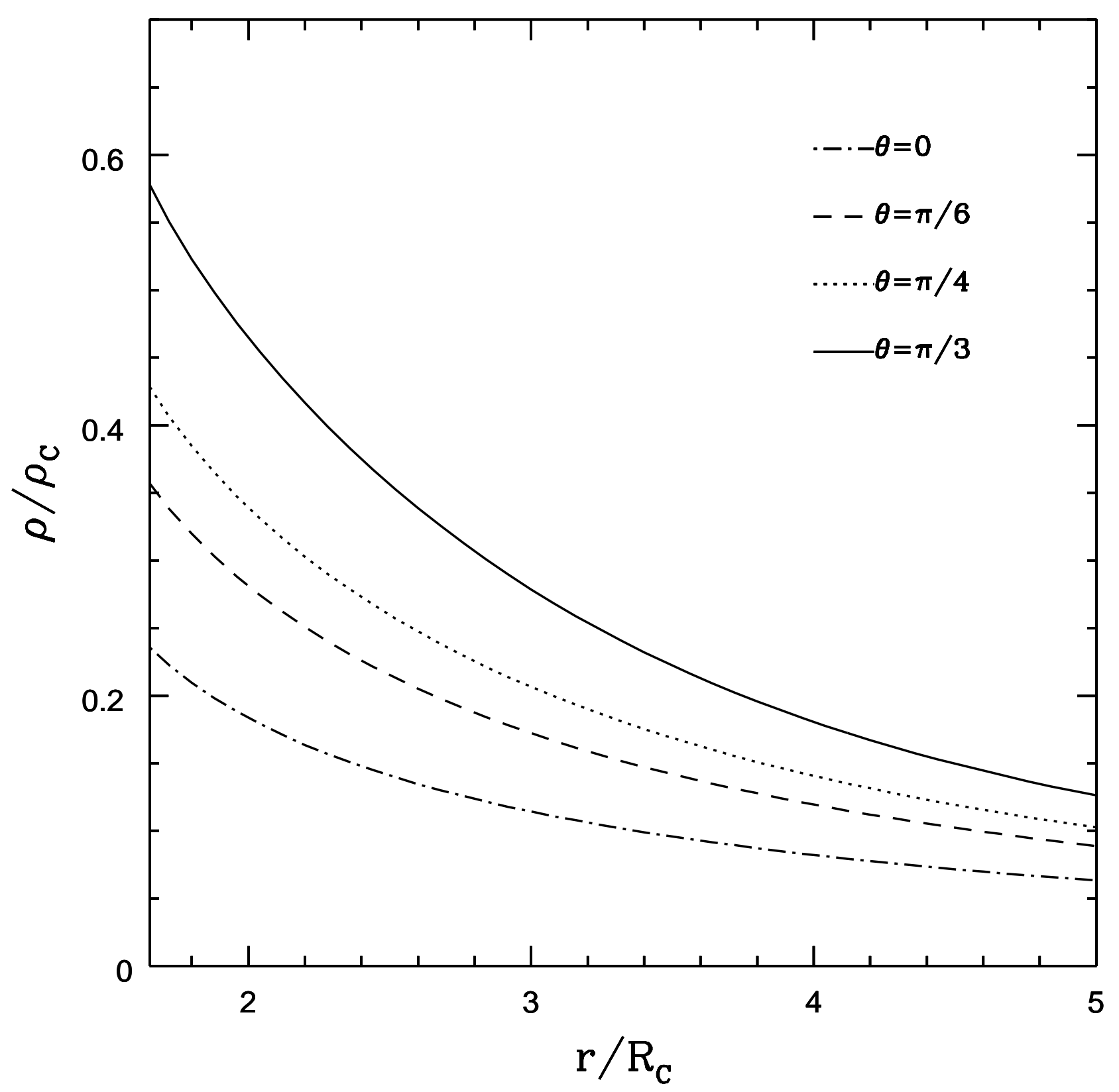




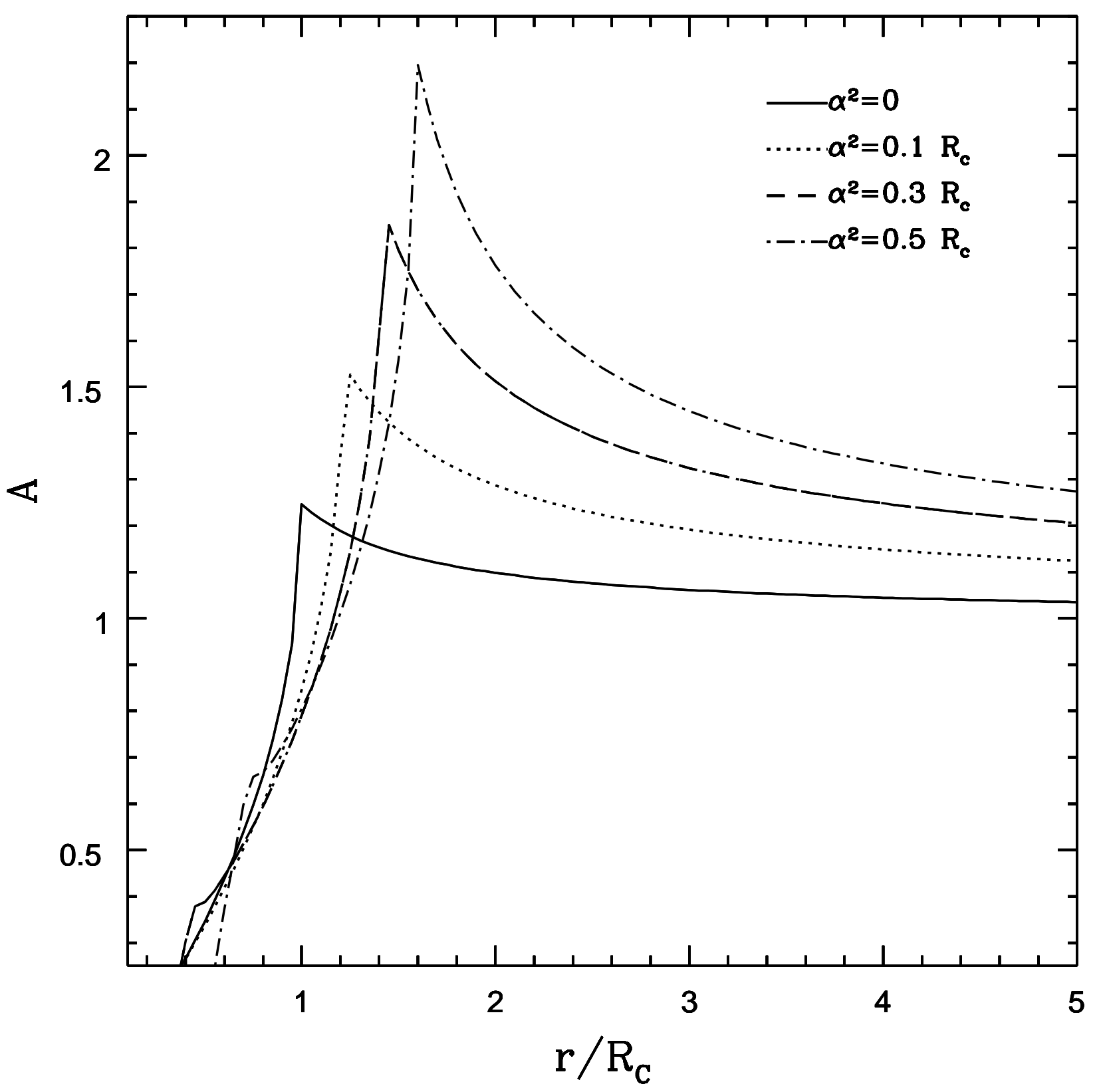




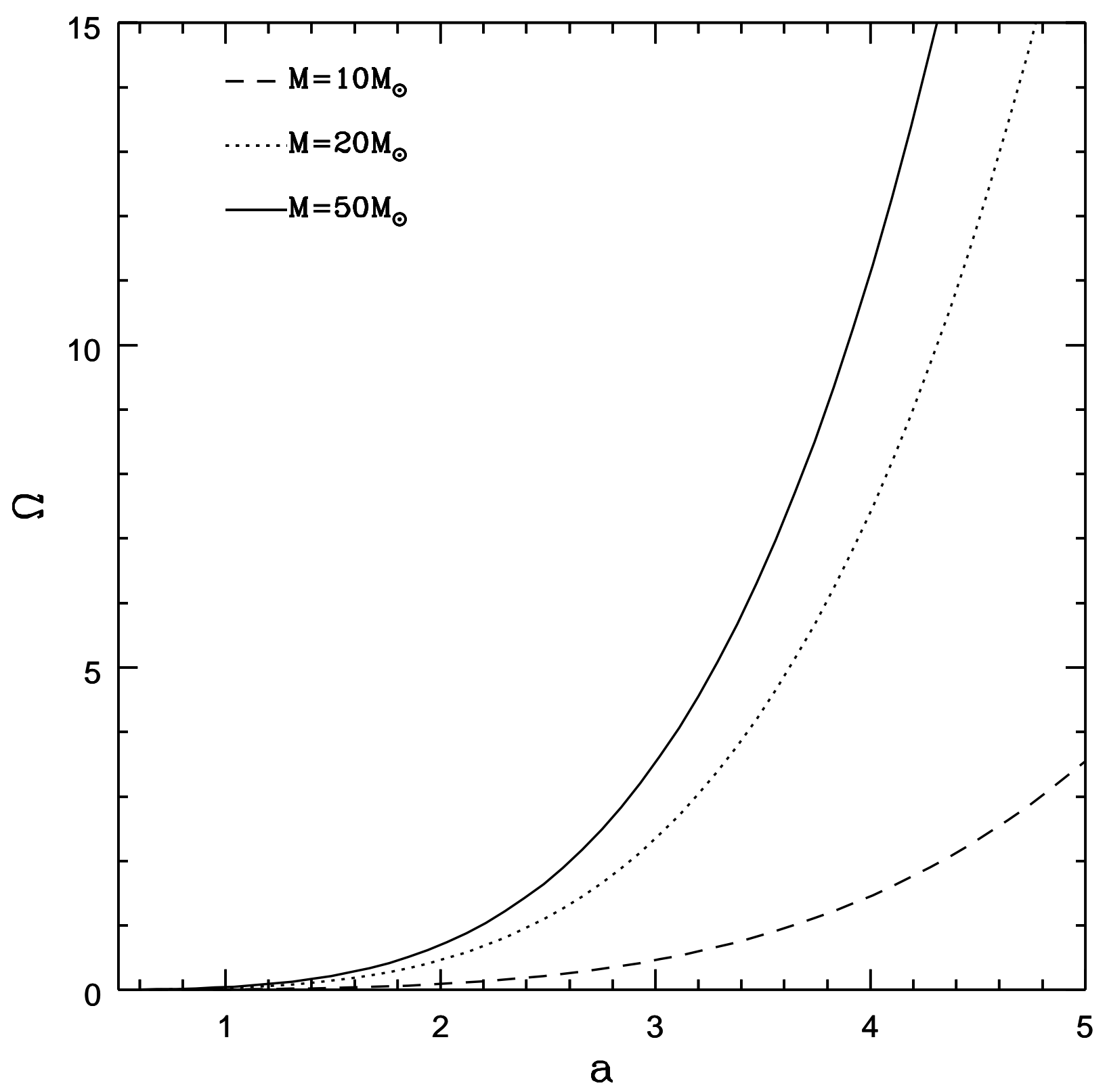




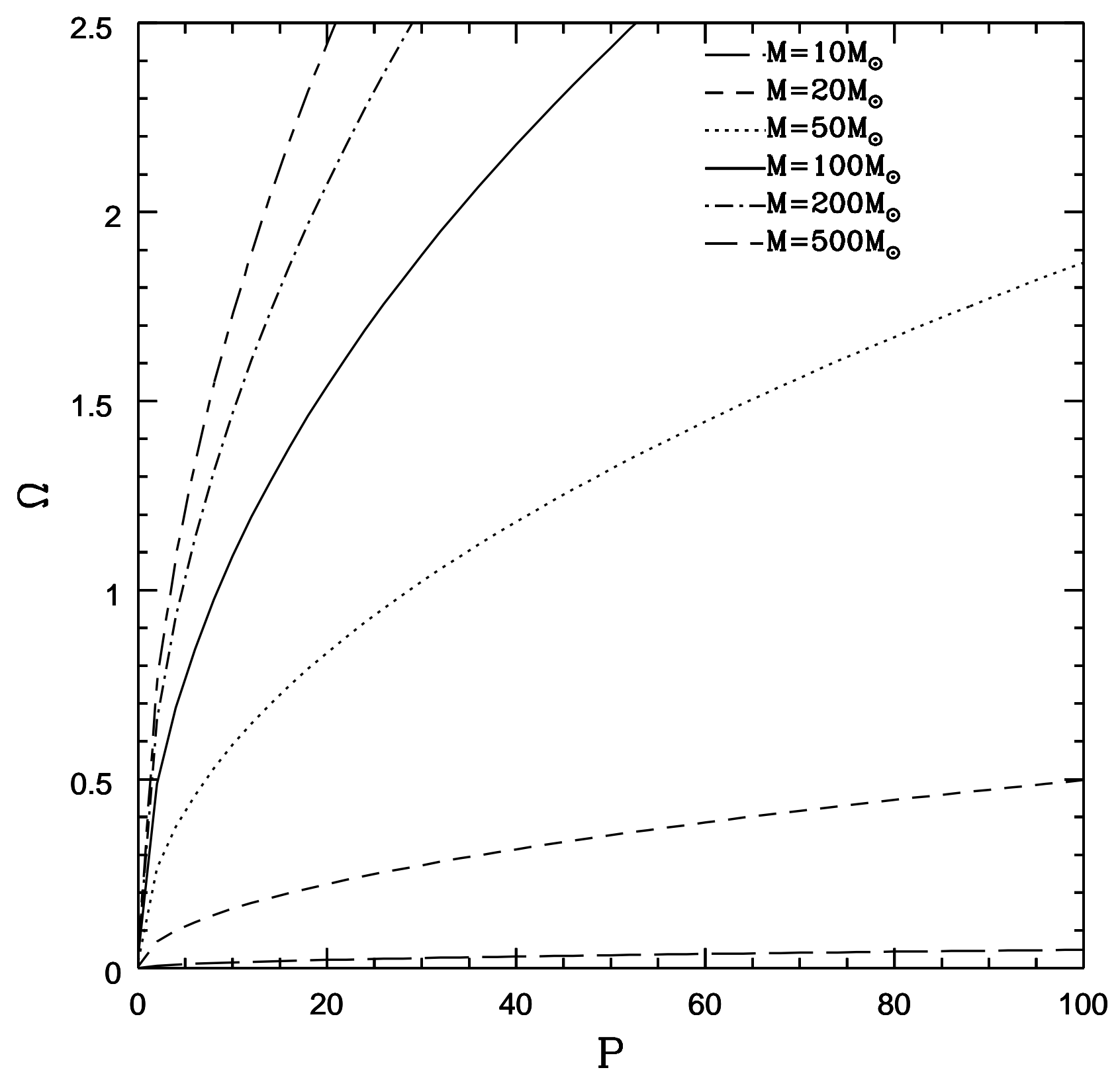




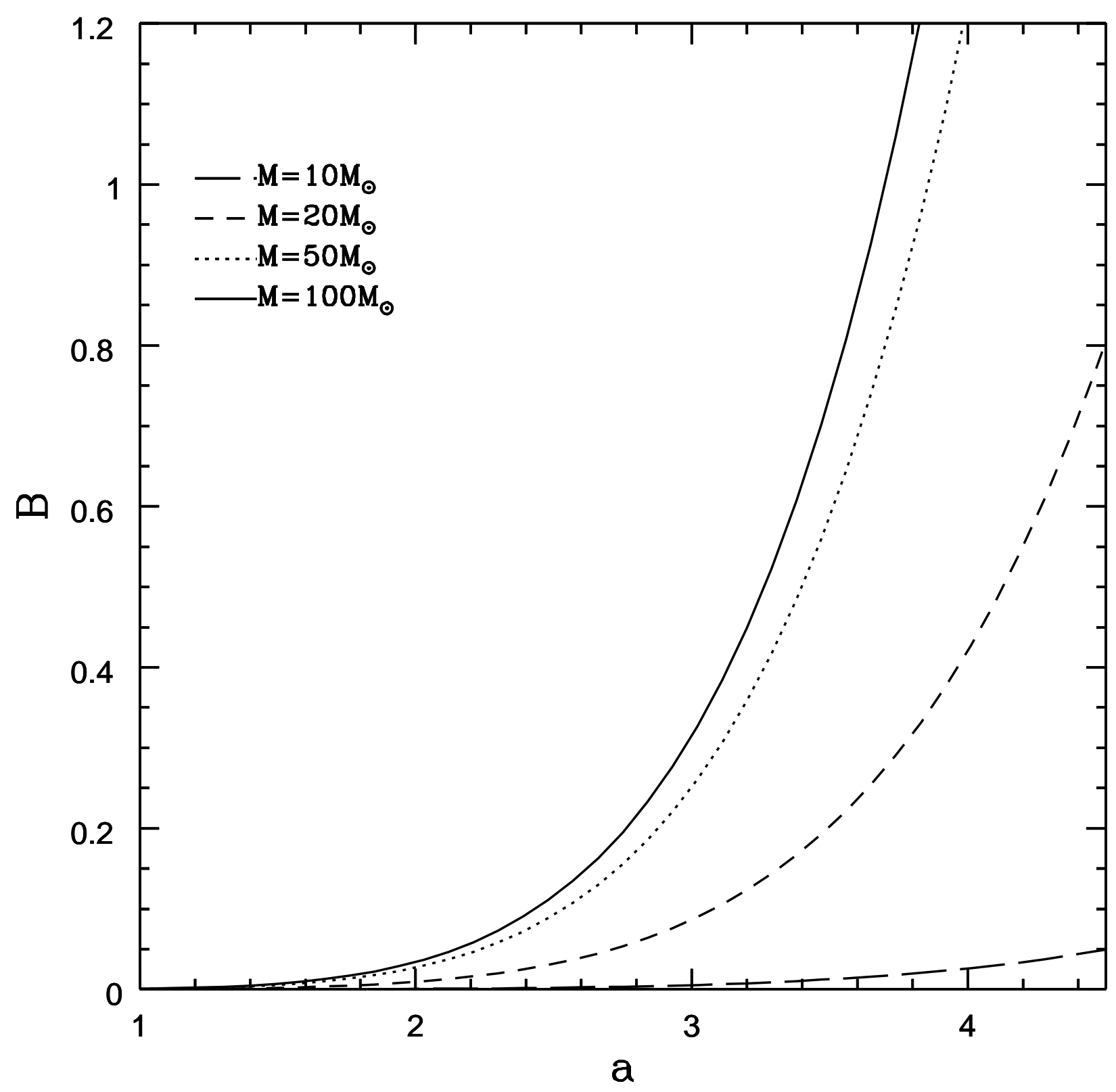




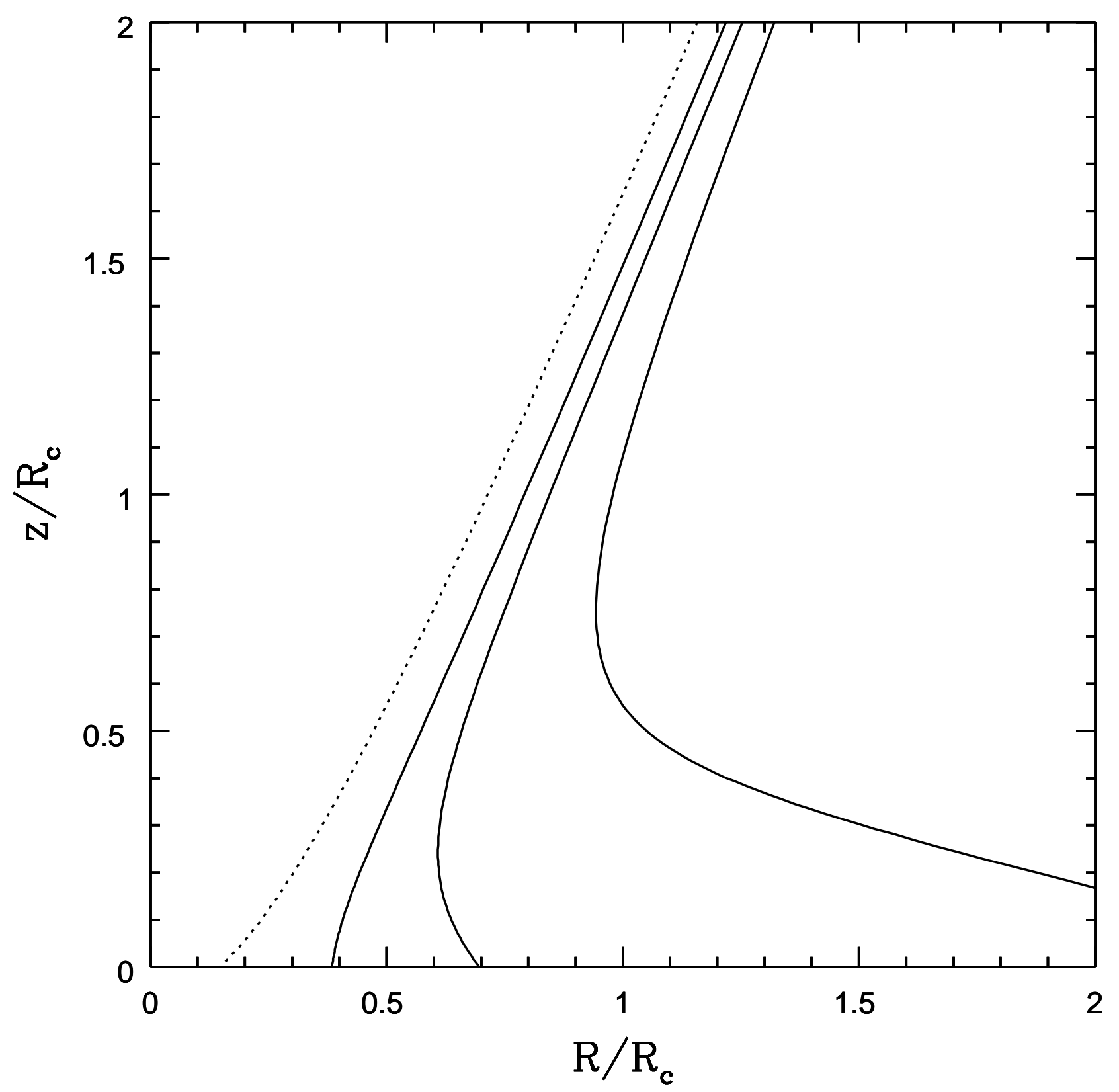

